TRANSACTIONS OF THE

AMERICAN MATHEMATICAL SOCIETY

Volume 355, Number 7 , Pages 2795-2812

S 0002-9947(03)03276-8

Article electronically published on March 12, 2003

\title{
LARGE RECTANGULAR SEMIGROUPS IN STONE-ČECH COMPACTIFICATIONS
}

\author{
NEIL HINDMAN, DONA STRAUSS, AND YEVHEN ZELENYUK
}

\begin{abstract}
We show that large rectangular semigroups can be found in certain Stone-Čech compactifications. In particular, there are copies of the $2^{\mathfrak{c}} \times 2^{\mathfrak{c}}$ rectangular semigroup in the smallest ideal of $(\beta \mathbb{N},+)$, and so, a semigroup consisting of idempotents can be embedded in the smallest ideal of $(\beta \mathbb{N},+)$ if and only if it is a subsemigroup of the $2^{\mathfrak{c}} \times 2^{\mathfrak{c}}$ rectangular semigroup. In fact, we show that for any ordinal $\lambda$ with cardinality at most $\mathfrak{c}, \beta \mathbb{N}$ contains a semigroup of idempotents whose rectangular components are all copies of the $2^{\mathfrak{c}} \times 2^{\mathfrak{c}}$ rectangular semigroup and form a decreasing chain indexed by $\lambda+1$, with the minimum component contained in the smallest ideal of $\beta \mathbb{N}$.

As a fortuitous corollary we obtain the fact that there are $\leq_{L}$-chains of idempotents of length $\mathfrak{c}$ in $\beta \mathbb{N}$. We show also that there are copies of the direct product of the $2^{\mathfrak{c}} \times 2^{\mathfrak{c}}$ rectangular semigroup with the free group on $2^{\mathfrak{c}}$ generators contained in the smallest ideal of $\beta \mathbb{N}$.
\end{abstract}

\section{INTRODUCTION}

The Stone-Čech compactification of the integers $\beta \mathbb{N}$ has a semigroup structure which extends addition on $\mathbb{N}$ and has significant applications in Ramsey Theory and topological dynamics. Some questions about the algebra of $\beta \mathbb{N}$, which sound deceptively simple, have been found to be extremely difficult. For example, it is not known whether $\beta \mathbb{N}$ contains any finite semigroups whose members are not all idempotent. Whether there were two idempotents in $\beta \mathbb{N}$ whose sum was an idempotent different from either remained an open question for several years. It was answered in the affirmative in [10, in which it was shown that a certain finite rectangular semigroup could be embedded in $\beta \mathbb{N}$. (A semigroup is rectangular if and only if it is isomorphic to the direct product of a left zero semigroup and a right zero semigroup. A rectangular component of a semigroup of idempotents is a maximal rectangular subsemigroup. As suggested by the name, distinct components are disjoint. The components are partially ordered by the relation $P \leq Q$ if and only if $P Q \subseteq P$, equivalently $Q P \subseteq P$ [7] Theorem 1].) In this paper, we show that the rectangular semigroup $2^{\mathfrak{c}} \times 2^{\mathfrak{c}}$, with the first factor being left zero and the second right zero, can be embedded in $\beta \mathbb{N}$. Indeed, $\beta \mathbb{N}$ contains semigroups of idempotents which are the union of $\mathfrak{c}$ rectangular components each isomorphic to $2^{\mathfrak{c}} \times 2^{\mathfrak{c}}$. We shall show also that if $S$ is an infinite cancellative semigroup with cardinality $\kappa$, then $\beta S \backslash S$ contains a semigroup of idempotents which is the union

Received by the editors April 12, 2002 and, in revised form, November 14, 2002.

2000 Mathematics Subject Classification. Primary 20M10; Secondary 22A15, 54H13.

The first author acknowledges support received from the National Science Foundation (USA) via grant DMS-0070593. 
of at least $\kappa$ rectangular components, each isomorphic to $2^{2^{\kappa}} \times 2^{2^{\kappa}}$, with the first factor being left zero and the second right zero.

We first review terminology used in the topological theory of semigroups. Let $S$ be a semigroup and a topological space. For each $s \in S$, we define mappings $\lambda_{s}$ and $\rho_{s}$ from $S$ to itself by $\lambda_{s}(t)=s t$ and $\rho_{s}(t)=t s$. $S$ is said to be a right topological semigroup if $\rho_{s}$ is continuous for every $s \in S$. In this case, the topological center $\Lambda(S)$ of $S$ is defined by $\Lambda(S)=\left\{s \in S: \lambda_{s}\right.$ is continuous $\}$. $S$ is said to be a semitopological semigroup if $\rho_{s}$ and $\lambda_{s}$ are both continuous for every $s \in S$. It is said to be a topological semigroup if the semigroup operation is a continuous mapping from $S \times S$ to $S$.

If $S$ is a discrete semigroup, we regard its Stone-Cech compactification $\beta S$ as the space of ultrafilters defined on $S$, with the topology defined by choosing the sets of the form $\bar{A}=\{p \in \beta S: A \in p\}$ as a base for the open sets. $\beta S$ is then a compact Hausdorff space and $\bar{A}=c \ell_{\beta S}(A)$. We regard $S$ as a subset of $\beta S$, by identifying each element of $S$ with the principal ultrafilter that it defines. $\beta S$ can be given a semigroup structure which extends the semigroup structure of $S$ in such a way that $\beta S$ is a compact right topological semigroup, with $S$ contained in its topological center. If $A \subseteq S, A^{*}$ will denote $\bar{A} \backslash A$.

We shall use basic algebraic properties that hold in all compact Hausdorff right topological semigroups. (We shall be assuming that all hypothesized topological spaces are Hausdorff.) A simple and important property is that every compact right topological semigroup $T$ contains an idempotent. $T$ has a smallest ideal $K(T)$, which is both the union of all the minimal left ideals and the union of all the minimal right ideals of $T$. Every right ideal of $T$ contains a minimal right ideal, and every left ideal of $T$ contains a minimal left ideal. If $L$ is a minimal left ideal and $R$ a minimal right ideal in $T$, then $R L=R \cap L$ is a group. So $R \cap L$ contains a unique idempotent. If $f: T \rightarrow T^{\prime}$ is a homomorphism from $T$ onto a compact right topological group $T^{\prime}$, then $f[K(T)]=K\left(T^{\prime}\right)$. For each minimal right ideal $R^{\prime}$ of $T^{\prime}$, there is a minimal right ideal $R$ of $T$ for which $f[R]=R^{\prime}$. The corresponding statement holds for left ideals as well. There are three natural orderings of the idempotents of $T$ defined by

$$
\begin{aligned}
e \leq_{L} f & \Leftrightarrow \quad e=e f, \\
e \leq_{R} f & \Leftrightarrow \quad e=f e, \text { and } \\
e \leq f & \Leftrightarrow \quad e f=f e=e .
\end{aligned}
$$

An idempotent $e$ is minimal with respect to any or all of these orderings if and only if $e \in K(T)$. The reader is referred to [1, 6], or 9] for proofs of these statements.

When $S$ is a discrete semigroup, the smallest ideal $K(\beta S)$ is of special importance for combinatorial applications, and in particular, the members of idempotents in $K(\beta S)$ have strong combinatorial properties. (See [6, Chapter 14].) Thus we are especially interested in those semigroups of idempotents that can be embedded in the smallest ideal of $\beta S$.

As we have already mentioned, a semigroup $S$ is rectangular provided it is isomorphic to the direct product of a left zero semigroup with a right zero semigroup. This is equivalent to saying that it satisfies the identities $x^{2}=x$ and $x y z=x z$. (The necessity is trivial. For the sufficiency, pick $x \in S$, note that $S x$ is a left zero semigroup, $x S$ is a right zero semigroup, and the function $(a, b) \mapsto a b$ from $S x \times x S$ to $S$ is an isomorphism.) We observe that a rectangular semigroup $S$ 
satisfies $S=K(S)=L R \sim L \times R$, where $L$ denotes any minimal left ideal and $R$ any minimal right ideal in $S$.

If $S$ is a semigroup, $E(S)$ will denote the set of idempotents in $S$.

If $A$ is any set, $\mathcal{P}_{f}(A)$ will denote the set of finite nonempty subsets of $A$.

\section{The Semigroups $\mathbb{H}_{\kappa}$}

The subsemigroup $\mathbb{H}=\bigcap_{n=1}^{\infty} c \ell_{\beta \mathbb{N}}\left(\mathbb{N} 2^{n}\right)$ of $(\beta \mathbb{N},+)$ holds all of the idempotents of $\beta \mathbb{N}$ and much of the known algebraic structure. (See [6, Section 6.1].) It occurs widely in the study of semigroups of the form $\beta S$. If $S$ is an infinite discrete cancellative semigroup, every $G_{\delta}$ subset of $S^{*}$ that contains an idempotent, contains copies of $\mathbb{H}[6$, Theorem 6.32]. $\mathbb{H}$ also has the property that any compact right topological semigroup with countable dense topological center is the image of $\mathbb{H}$ under a continuous homomorphism [6, Theorem 6.4]. In this section we introduce a semigroup $\mathbb{H}_{\kappa}$ which satisfies a similar conclusion for an arbitrary infinite cardinal $\kappa$. As a consequence of the results of the next section we shall conclude that each $\mathbb{H}_{\kappa}$ contains large rectangular subsemigroups.

Definition 2.1. Let $\kappa$ be an infinite cardinal. Then $W_{\kappa}=\bigoplus_{\alpha<\kappa} \mathbb{Z}_{2}$. For $x \in W_{\kappa}$, $\operatorname{supp}(x)=\left\{\alpha<\kappa: x_{\alpha} \neq 0\right\}$. For $\alpha<\kappa, e_{\alpha}$ is that member of $W_{\kappa}$ such that $\operatorname{supp}\left(e_{\alpha}\right)=\{\alpha\}$, and

$$
\mathbb{H}_{\kappa}=\bigcap_{\alpha<\kappa} c \ell_{\beta W_{\kappa}}\left\{x \in W_{\kappa} \backslash\{\overline{0}\}: \min \operatorname{supp}(x) \geq \alpha\right\} .
$$

The structure of $\mathbb{H}_{\omega}$ is that induced by an "oid" as introduced by John Pym 8 . When we say that two structures are "topologically and algebraically isomorphic", we mean that there is one function between them that is both an isomorphism and a homeomorphism.

Theorem 2.2. The compact right topological semigroups $\mathbb{H}$ and $\mathbb{H}_{\omega}$ are topologically and algebraically isomorphic.

Proof. [6] Theorem 6.15].

It is a fact [6, Lemma 6.8] that all of the idempotents of $\beta \mathbb{N}$ are in $\mathbb{H}$. Thus, by [6. Theorem 1.65], $K(\mathbb{H})=K(\beta \mathbb{N}) \cap \mathbb{H}$.

Theorem 2.3. Let $S$ be a countably infinite discrete group. Then $\beta S \backslash S$ contains a topological and algebraic copy $T$ of $\mathbb{H}$ such that $K(T)=K(\beta S) \cap T$.

Proof. Take any idempotent $p \in K(\beta S)$. By [6, Theorem 9.13], there is a left invariant zero-dimensional Hausdorff topology on $S$ in which the ultrafilter $p$ converges to 1 . Then by [6, Theorem 7.24], with $X=G=V(a)=S$ for every $a \in G$, there is a topological and algebraic embedding $f: \mathbb{H} \rightarrow \beta S \backslash S$ such that $p \in f[\mathbb{H}]$. It remains to apply [6, Theorem 1.65].

A similar result applies to the semigroup $(\mathbb{N}, \cdot)$. Given $n \in \omega$ we define the binary support of $n$ by $n=\sum_{t \in \operatorname{supp}_{2}(n)} 2^{t}$ and $\operatorname{supp}_{2}(0)=\emptyset$.

Theorem 2.4. Let $S=(\mathbb{N}, \cdot)$. There is a topological and algebraic copy $T$ of $\mathbb{H}$ contained in $\beta S \backslash S$ which contains all of the idempotents of $\beta S \backslash S$. In particular, $K(T)=T \cap K(\beta S)$. 
Proof. Let $\left\langle p_{i}\right\rangle_{i=1}^{\infty}$ be the sequence of primes. Then $\left(\bigoplus_{i=1}^{\infty} \omega,+\right)$ is isomorphic to $(\mathbb{N}, \cdot)$ via the map $x \mapsto \prod_{i=1}^{\infty} p_{i}^{x_{i}}$; so we shall take $S$ to be $\bigoplus_{i=1}^{\infty} \omega$. For each $i \in \mathbb{N}$, let $\pi_{i}: S \rightarrow \omega$ be the projection to the $i^{\text {th }}$ factor and let $\widetilde{\pi}_{i}: \beta S \rightarrow \beta \omega$ be its continuous extension.

Let $\left\{X_{i}: i \in \mathbb{N}\right\}$ be a partition of $\omega$ into infinite sets and for each $i \in \mathbb{N}$, let $\phi_{i}: X_{i} \rightarrow \omega$ be a bijection. We define $\theta: \omega \rightarrow S$ by agreeing that for each $i \in \mathbb{N}$ and each $n \in \omega$,

$$
\pi_{i}(\theta(n))=\sum_{j \in \operatorname{supp}_{2}(n) \cap X_{i}} 2^{\phi_{i}(j)},
$$

where $\sum_{j \in \emptyset} 2^{\phi_{i}(j)}=0$. We note that $\theta$ is a bijection. (If $j \in \operatorname{supp}_{2}(n) \backslash \operatorname{supp}_{2}(m)$, then for some $i, j \in X_{i}$ and so $\phi_{i}(j) \in \operatorname{supp}_{2}\left(\pi_{i}(n)\right) \backslash \operatorname{supp}_{2}\left(\pi_{i}(m)\right)$. Also, given $x \in S$, for each $i \in \mathbb{N}$ let $Y_{i}=\phi_{i}{ }^{-1}\left[\operatorname{supp}_{2}\left(\pi_{i}(x)\right)\right]$, let $Z=\bigcup_{i \in \mathbb{N}} Y_{i}$, and let $n=\sum_{t \in Z} 2^{t}$. Then $\theta(n)=x$.) Consequently by [6, Exercise 3.4.1] the continuous extension $\widetilde{\theta}: \beta \omega \rightarrow \beta S$ of $\theta$ is a bijection. Since $\theta(n+m)=\theta(n)+\theta(m)$ if $\operatorname{supp}_{2}(n) \cap \operatorname{supp}_{2}(m)=\emptyset, \widetilde{\theta}$ is a homomorphism on $\mathbb{H}$ by [6, Lemma 6.3].

To complete the proof, let $p$ be an idempotent in $\beta S \backslash S$. Since

$$
\widetilde{\theta}[\mathbb{H}]=\widetilde{\theta}\left[\bigcap_{n=1}^{\infty} \overline{\omega 2^{n}}\right] \backslash\{0\}=\left(\bigcap_{n=1}^{\infty} \overline{\theta\left[\omega 2^{n}\right]} \backslash\{0\},\right.
$$

it suffices to show that for all $n \in \mathbb{N}, \theta\left[\omega 2^{n}\right] \in p$. So let $n \in \mathbb{N}$ and suppose that $\theta\left[\omega 2^{n}\right] \notin p$. Pick $t \in\left\{1,2, \ldots, 2^{n}-1\right\}$ such that $\theta\left[\omega 2^{n}+t\right] \in p$, pick $j \in \operatorname{supp}_{2}(t)$, and pick $i$ such that $j \in X_{i}$. Now $\widetilde{\pi}_{i}(p)$ is an idempotent; so either $\widetilde{\pi}_{i}(p)=0$ or $\widetilde{\pi}_{i}(p) \in \beta \mathbb{N} \backslash \mathbb{N}$. Thus by [6] Lemma 6.6] $\omega 2^{\phi_{i}(j)+1} \in p$. Pick $x \in \omega 2^{\phi_{i}(j)+1} \cap$ $\pi_{i}\left[\theta\left[\omega 2^{n}+t\right]\right]$ and pick $k \in \omega 2^{n}+t$ such that $x=\pi_{i}(\theta(k))$. Then $j \in \operatorname{supp}_{2}(k) \cap X_{i}$; so $\phi_{i}(j) \in \operatorname{supp}_{2}(x)$, contradicting the fact that $x \in \omega 2^{\phi_{i}(j)+1}$.

Observe that if $\kappa>\omega$, then $\mathbb{H}_{\kappa} \cap K\left(\beta W_{\kappa}\right)=\emptyset$. To see this, one lets $p \in \mathbb{H}_{\kappa}$ and

$$
q \in \bigcap_{k<\omega} c l\left\{x \in W_{\kappa} \backslash\{\overline{0}\}: \min \operatorname{supp}(x) \geq k \text { and } \max \operatorname{supp}(x)<\omega\right\} .
$$

Then $p \notin \beta W_{\kappa}+q+p$ and so [6, Theorem 4.39] applies.

Theorem 2.5. Let $\kappa$ be an infinite cardinal and let $T$ be a compact right topological semigroup. Assume that there is a set $A \subseteq \Lambda(T)$ such that $|A| \leq \kappa$ and $A$ is dense in $T$. Then there is a continuous surjective homomorphism $f: \mathbb{H}_{\kappa} \rightarrow T$.

Proof. Enumerate $A$ as $\left\{t_{\alpha}: \alpha<\kappa\right\}$, with repetition if $|A|<\kappa$. Let $\left\{I_{\gamma}: \gamma<\kappa\right\}$ be a partition of $\kappa$ into subsets of size $\kappa$. Define $h: W_{\kappa} \rightarrow T$ by first agreeing that for each $\alpha<\kappa, h\left(e_{\alpha}\right)=t_{\gamma}$, where $\alpha \in I_{\gamma}$. Then for $F \in \mathcal{P}_{f}(\kappa)$, define $h\left(\sum_{\alpha \in F} e_{\alpha}\right)=\prod_{\alpha \in F} h\left(e_{\alpha}\right)$, where the product is taken in increasing order of indices. Define $h(0)$ arbitrarily. Let $\widetilde{h}: \beta W_{\kappa} \rightarrow T$ be the continuous extension of $h$ and let $f$ be the restriction of $\widetilde{h}$ to $\mathbb{H}_{\kappa}$.

To see that $h\left[\mathbb{H}_{\kappa}\right]=T$, it suffices to show that $A \subseteq h\left[\mathbb{H}_{\kappa}\right]$. Given $\gamma<\kappa$, we have that $\left|I_{\gamma}\right|=\kappa$. Pick a $\kappa$-uniform ultrafilter $p$ on $\left\{e_{\alpha}: \alpha \in I_{\gamma}\right\}$. Then $p \in \mathbb{H}_{\kappa}$ and $h(p)=t_{\gamma}$ because $f$ is constantly equal to $t_{\gamma}$ on $\left\{e_{\alpha}: \alpha \in I_{\gamma}\right\}$.

To see that $f$ is a homomorphism it suffices by [6, Theorem 4.21] to observe that whenever $x \in W_{\kappa} \backslash\{\overline{0}\}$ and $y \in W_{\kappa} \backslash\{\overline{0}\}$ with $\min \operatorname{supp}(y)>\max \operatorname{supp}(x)$, then $h(x+y)=h(x) \cdot h(y)$.

Definition 2.6. Let $S$ be a semigroup, let $\kappa$ be a cardinal, and let $\left\langle t_{\lambda}\right\rangle_{\lambda<\kappa}$ be a $\kappa$-sequence in $S$.

(a) Given $F \in \mathcal{P}_{f}(\kappa), \prod_{\lambda \in F} t_{\lambda}$ is the product in increasing order of indices. 
(b) If $D \subseteq \kappa$, then $F P\left(\left\langle t_{\lambda}\right\rangle_{\lambda \in D}\right)=\left\{\prod_{\lambda \in F} t_{\lambda}: F \in \mathcal{P}_{f}(D)\right\}$.

(c) The sequence $\left\langle t_{\lambda}\right\rangle_{\lambda<\kappa}$ has distinct finite products if and only if whenever $F, G \in \mathcal{P}_{f}(\lambda)$ and $\prod_{\lambda \in F} t_{\lambda}=\prod_{\lambda \in G} t_{\lambda}$, one must have $F=G$.

Theorem 2.7. Let $S$ be an infinite cancellative discrete semigroup with cardinality $\kappa$. Then $\beta S \backslash S$ contains a topological and algebraic copy of $\mathbb{H}_{\kappa}$.

Proof. By [6, Lemma 6.31], we may choose a $\kappa$-sequence $\left\langle t_{\lambda}\right\rangle_{\lambda<\kappa}$ in $S$ with distinct finite products. Let $T=F P\left(\left\langle t_{\lambda}\right\rangle_{\lambda<\kappa}\right)$. For each $\gamma<\kappa$, let $T_{\gamma}=F P\left(\left\langle t_{\lambda}\right\rangle_{\gamma<\lambda<\kappa}\right)$. We put $\widetilde{T}=\bigcap_{\gamma<\kappa} c l_{\beta S}\left(T_{\gamma}\right)$. By [6] Theorem 4.20], $\widetilde{T}$ is a subsemigroup of $\beta S$.

We define $\theta: T \rightarrow W_{\kappa}$ by $\theta\left(\prod_{\lambda \in F} t_{\lambda}\right)=\sum_{\lambda \in F} e_{\lambda}$. (Since the sequence $\left\langle t_{\lambda}\right\rangle_{\lambda<\kappa}$ has distinct finite products, the function $\theta$ is well defined.) Let $\widetilde{\theta}: c \ell_{\beta S}(T) \rightarrow \beta W_{\kappa}$ denote the continuous extension of $\theta$. By [6, Theorem 4.21], the restriction of $\widetilde{\theta}$ to $\widetilde{T}$ is a homomorphism. Now $\widetilde{\theta}$ is injective, by [6] Exercise 3.4.1]. Since for each $\gamma<\kappa, \widetilde{\theta}\left[T_{\gamma}\right]=c \ell_{\beta W_{\kappa}}\left\{x \in W_{\kappa}: \min \operatorname{supp}(x)>\gamma\right\}, \widetilde{\theta}$ maps $\widetilde{T}$ onto $\mathbb{H}_{\kappa}$. Thus $\tilde{\theta}$ determines an isomorphism from $\widetilde{T}$ onto $\mathbb{H}_{\kappa}$.

\section{Chains of ReCtangular semigroups in $\mathbb{H}_{\kappa}$}

Let $\kappa$ be an infinite cardinal and let $V_{\kappa}$ denote the rectangular semigroup $\kappa \times \kappa$, with the first factor being left zero and the second right zero. We show in this section (in Corollary 3.10 ) that for any infinite cardinal $\kappa$, algebraic copies of $V_{2^{2^{\kappa}}}$ can be found in $K\left(\mathbb{H}_{\kappa}\right)$. Indeed, if $\lambda$ is any ordinal for which $|\lambda| \leq \kappa$, there is a decreasing chain $\left\langle D_{p}\right\rangle_{p \leq \lambda}$ of disjoint copies of $V_{2^{2^{\kappa}}}$ contained in $\mathbb{H}_{\kappa}$, with $D_{\lambda}$ embedded in $K\left(\mathbb{H}_{\kappa}\right)$.

Notice that $V_{\kappa}$ contains a copy of every rectangular semigroup of cardinality at most $\kappa$.

Definition 3.1. Let $\lambda$ be any ordinal and let $A$ be any nonempty set. Let 0 denote a selected element of $A$. For $p<\lambda$, let $C_{p}=A \times A \times\{p\}$ and let

$$
C=C_{A, \lambda}=A \cup \bigcup_{p<\lambda} C_{p}=A \cup(A \times A \times \lambda) .
$$

The operation - on $C$ is defined as follows. Let $a, b, c, d \in A$ and let $p, q<\lambda$. Then

$$
\begin{aligned}
a \cdot b & =b, \\
a \cdot(b, c, p) & =(b, c, p), \\
(b, c, p) \cdot a & =(b, a, p), \\
(a, b, p) \cdot(c, d, q) & =(a, d, p \vee q),
\end{aligned}
$$

where $p \vee q$ is the maximum of $p$ and $q$.

We leave to the reader the routine verification that the operation on $C_{A, \lambda}$ is associative. Notice that for any $p<\lambda, C_{p}$ is a copy of $V_{|A|}$.

Definition 3.2. Let $\lambda$ be an ordinal and let $p<\lambda$. We let $u_{p}=(0,0, p)$, and for every $x=(a, b, p) \in C_{p}$, we let $x_{\ell}=(a, 0,0)$ and $x_{r}=b$. For $x \in A$, we let $x_{\ell}=x_{r}=x$.

The following is simple, and its proof is like that of [6, Theorem 1.46].

Lemma 3.3. Let $S$ be a semigroup, let $H$ be an ideal of $S$, let $L$ be a minimal left ideal of $H$, let $R$ be a minimal right ideal of $H$, and let $x \in S$. Then $L x$ is a minimal left ideal of $H, x R$ is a minimal right ideal of $H, x L \subseteq L$, and $R x \subseteq R$. 
Proof. It suffices to establish the assertions about $L x$ and $x L$. Now $L x \subseteq H x \subseteq H$ and $H L x \subseteq L x$; so $L x$ is a left ideal of $H$. Let $M$ be a left ideal of $H$ with $M \subseteq L x$. Let $J=\{y \in L: y x \in M\}$. Given $y \in J$ and $z \in H$, we have $z y \in L$ and $z y x \in M$; so $z y \in J$. Thus $J=L$ and so $M=L x$.

Next, given $y \in L=H y$, so pick $z \in H$ such that $y=z y$. Then $x y=x z y \in$ $H y=L$.

Lemma 3.4. Let $A$ be a nonempty set with distinguished element 0 , and let $C=$ $C_{A, 1}$. Let $T$ be a right topological semigroup, and let $f: T \rightarrow C$ be a surjective homomorphism for which $f^{-1}[A]$ and $f^{-1}\left[C_{0}\right]$ are compact. Then there is a homomorphism $g: C \rightarrow T$ such that $f \circ g$ is the identity on $C$ and $g\left[C_{0}\right] \subseteq K\left(f^{-1}\left[C_{0}\right]\right)$. If $T$ is compact, then $g\left[C_{0}\right] \subseteq K(T)$.

Proof. We first define $g$ on $A$. We have that $f^{-1}[A]$ is a compact semigroup. Choose a minimal right ideal $N$ of $f^{-1}[A]$. For each $a \in A, f^{-1}[\{a\}]$ is a left ideal of $f^{-1}[A]$. So choose a minimal left ideal $S_{a}$ of $f^{-1}[A]$ with $S_{a} \subseteq f^{-1}[\{a\}]$, and let $g(a)$ be the identity of the group $N \cap S_{a}$. Then immediately $f(g(a))=a$. Also, given $a, b \in A$ we have that $g(a)$ and $g(b)$ are idempotents in $N$; so $g(a) g(b)=g(b)=g(a b)$.

Let $B=\{(a, 0,0): a \in A\}$. Then $B$ is a left ideal of $C_{0}$; so $f^{-1}[B]$ is a left ideal of $f^{-1}\left[C_{0}\right]$ which therefore contains a minimal left ideal $L$ of $f^{-1}\left[C_{0}\right]$. For each $a \in A$ let $F_{a}=\{(a, b, 0): b \in A\}$. Then $F_{a}$ is a right ideal of $C_{0}$. So pick a minimal right ideal $R_{a}$ of $f^{-1}\left[C_{0}\right]$ with $R_{a} \subseteq f^{-1}\left[F_{a}\right]$. By Lemma [3.3] since $f^{-1}\left[C_{0}\right]$ is an ideal of $f^{-1}\left[A \cup C_{0}\right]$, we have that $g(0) \cdot R_{a}$ is a minimal right ideal of $f^{-1}\left[C_{0}\right]$ and $L \cdot g(a)$ is a minimal left ideal of $f^{-1}\left[C_{0}\right]$. For $a, b \in A$, let $g(a, b, 0)$ be the identity of the group $g(0) \cdot R_{a} \cdot L \cdot g(b)$. Notice that if $T$ is compact, then $K(T) \subseteq f^{-1}\left[C_{0}\right]$ so that $K\left(f^{-1}\left[C_{0}\right]\right) \subseteq K(T)$ and thus $g(a, b, 0) \in K(T)$. Also $g(a, b, 0)=g(0) \cdot x \cdot y \cdot g(b)$ for some $x \in R_{a}$ and some $y \in L$. So $f(g(a, b, 0))=0 \cdot f(x) \cdot f(y) \cdot b=(a, b, 0)$.

To conclude the proof we need to show that $g$ is a homomorphism. First we let $a, b, c \in A$ and show that $g(a) \cdot g(b, c, 0)=g(b, c, 0)$ and $g(b, c, 0) \cdot a=g(b, a, 0)$. Pick $x \in R_{b} \cdot L$ such that $g(b, c, 0)=g(0) \cdot x \cdot g(c)$. Then

$$
\begin{aligned}
g(a) \cdot g(b, c, 0) & =g(a) \cdot g(0) \cdot x \cdot g(c) \\
& =g(0) \cdot x \cdot g(c) \\
& =g(b, c, 0) .
\end{aligned}
$$

So the first claim holds directly. Multiplying on the left by $g(b, c, 0)$ and on the right by $g(a)$ one sees that $g(b, c, 0) \cdot g(a)$ is idempotent. Since $g(b, c, 0) \cdot g(a) \in$ $g(0) \cdot R_{b} \cdot L \cdot g(c) \cdot g(a)=g(0) \cdot R_{b} \cdot L \cdot g(a)$, we must have that $g(b, c, 0) \cdot g(a)$ is the identity of $g(0) \cdot R_{b} \cdot L \cdot g(a)$, namely $g(b, a, 0)$.

Finally, let $a, b, c, d \in A$. Then $g(a, b, 0) \cdot g(c, d, 0) \in g(0) \cdot R_{a} \cdot L \cdot g(b) \cdot g(0) \cdot$ $R_{c} \cdot L \cdot g(d) \subseteq g(0) \cdot R_{a} \cdot L \cdot g(d)$. So it suffices to show that $g(a, b, 0) \cdot g(c, d, 0)$ is idempotent. These elements satisfy $g(a, 0,0) \in L \cdot g(0)$ and $g(c, 0,0) \in L$. $g(0)$. So as idempotents in the same minimal left ideal of $f^{-1}\left[C_{0}\right]$, we have that $g(a, 0,0) \cdot g(c, 0,0)=g(a, 0,0)$. Recall that we have shown that for any $x, y \in A$, $g(0) \cdot g(x, y, 0)=g(x, y, 0)$ and $g(x, y, 0) \cdot g(0)=g(x, 0,0)$. Thus we have

$$
\begin{aligned}
g(a, b, 0) \cdot g(c, d, 0) \cdot g(a, b, 0) & =g(a, b, 0) \cdot g(0) \cdot g(c, d, 0) \cdot g(0) \cdot g(a, b, 0) \\
& =g(a, 0,0) \cdot g(c, 0,0) \cdot g(a, b, 0) \\
& =g(a, 0,0) \cdot g(a, b, 0) \\
& =g(a, b, 0) \cdot g(0) \cdot g(a, b, 0) \\
& =g(a, b, 0) \cdot g(a, b, 0)=g(a, b, 0) .
\end{aligned}
$$


Multiplying on the right by $g(c, d, 0)$ we have that $g(a, b, 0) \cdot g(c, d, 0)$ is idempotent.

We now consider the situation in which $\lambda>1$. For $\lambda>\omega$ we do not necessarily get that $g$ is a homomorphism, but we come close.

Theorem 3.5. Let $A$ be a nonempty set with distinguished element 0 , let $\lambda$ be an ordinal, and let $C=C_{A, \lambda}$. Let $T$ be a right topological semigroup, and $f: T \rightarrow C$ be a surjective homomorphism such that $f^{-1}[A]$ is compact and $f^{-1}\left[C_{p}\right]$ is compact for every $p<\lambda$. Then there is a function $g: C \rightarrow T$ such that $f \circ g$ is the identity and $g$ has the following properties:

(i) If $q \leq p<\lambda, x \in C_{p}$, and $y \in A \cup C_{q}$, then $g(x y)=g(x) \cdot g(y)$ and $g(y) \cdot g(x)$ is an idempotent in the same minimal left ideal of $f^{-1}\left[C_{p}\right]$ as $g(y x)$.

(ii) If $p<\lambda, x \in C_{p}$, and $y \in A \cup C_{0}$, then $g(y) \cdot g(x)=g(y x)$.

(iii) If $q \leq p<\lambda, n \in \omega, p=q+n, y \in C_{q}$, and $x \in C_{p}$, then $g(y) \cdot g(x)=g(y x)$.

(iv) If $p<\lambda$, then $g\left[C_{p}\right] \subseteq K\left(f^{-1}\left[C_{p}\right]\right)$.

(v) If $T$ is compact and $\lambda$ is a successor, then $g\left[C_{\lambda-1}\right] \subseteq K(T)$.

The semigroup $T$ contains a semigroup $D=\bigcup_{p<\lambda} D_{p}$ of idempotents where each $D_{p}$ is a rectangular component of $D$ with $g\left[C_{p}\right] \subseteq D_{p}$ and the sequence $\left\langle D_{p}\right\rangle_{p<\lambda}$ is decreasing in the ordering of components, so that for each $p<\lambda, D_{p}=K\left(\bigcup_{q \leq p} D_{q}\right)$. If $|A| \geq|\lambda| \geq \omega$, then for each $p<\lambda,\left|D_{p}\right|$ is isomorphic to $V_{|A|}$.

Proof. For $p<\lambda$ we define $g$ on $A \cup \bigcup_{q \leq p} C_{q}$ by induction on $p$ so that $g$ satisfies conclusions (i), (ii), (iii), and (iv). By Lemma 3.4 we may define $g$ on $A \cup C_{0}$ so that $g$ satisfies (iv) and is a homomorphism and therefore satisfies (i), (ii), and (iii). Now let $0<p<\lambda$ and assume that $g$ has been defined on $A \cup \bigcup_{q<p} C_{q}$.

We show first that we may choose a minimal left ideal $L$ of $f^{-1}\left[C_{p}\right]$ such that $L \subseteq \bigcap_{q<p} f^{-1}\left[C_{p}\right] \cdot g\left(u_{q}\right)$ and $f[L]=C_{p} \cdot u_{p}$. A simple computation establishes that for each $q<p, f^{-1}\left[C_{p}\right] \cdot g\left(u_{q}\right)$ is a compact left ideal of $f^{-1}\left[C_{p}\right]$. Also, if $r<q<p$, then $f^{-1}\left[C_{p}\right] \cdot g\left(u_{q}\right) \subseteq f^{-1}\left[C_{p}\right] \cdot g\left(u_{r}\right)$. To see this, let $x \in f^{-1}\left[C_{p}\right]$. Then

$$
\begin{aligned}
x \cdot g\left(u_{q}\right) & =x \cdot g\left(u_{q} \cdot u_{r}\right) \\
& =x \cdot g\left(u_{q}\right) \cdot g\left(u_{r}\right) \quad \text { by }(\mathrm{i}) \\
& \in f^{-1}\left[C_{p}\right] \cdot g\left(u_{r}\right) .
\end{aligned}
$$

Consequently, $\bigcap_{q<p} f^{-1}\left[C_{p}\right] \cdot g\left(u_{q}\right)$ is a left ideal of $f^{-1}\left[C_{p}\right]$ and thus contains a minimal left ideal $L$ of $f^{-1}\left[C_{p}\right]$. Then $f[L]$ is a minimal left ideal of $C_{p}$.

Now given $x \in L$, one has $x \in f^{-1}\left[C_{p}\right] \cdot g\left(u_{0}\right)$. So for some $a \in A, f(x)=$ $(a, 0, p) \in C_{p} \cdot u_{p}$. Thus $f[L] \subseteq C_{p} \cdot u_{p}$. Since $C_{p} \cdot u_{p}$ is a minimal left ideal of $C_{p}$, we have $f[L]=C_{p} \cdot u_{p}$ as claimed.

If $p$ is a successor ordinal, observe that $g\left(u_{p-1}\right) \cdot f^{-1}\left[C_{p}\right]$ is a right ideal of $f^{-1}\left[C_{p}\right]$ and pick a minimal right ideal $R$ of $f^{-1}\left[C_{p}\right]$ with $R \subseteq g\left(u_{p-1}\right) \cdot f^{-1}\left[C_{p}\right]$. Then $f[R]$ is a minimal right ideal of $C_{p}$ and $f[R] \subseteq u_{p-1} \cdot C_{p}$; so $f[R]=u_{p-1} \cdot C_{p}$.

If $p$ is a limit ordinal, note that $f^{-1}\left[u_{p} \cdot C_{p}\right]$ is a right ideal of $f^{-1}\left[c_{p}\right]$. So pick a minimal right ideal $R$ of $f^{-1}\left[c_{p}\right]$ with $R \subseteq f^{-1}\left[u_{p} \cdot C_{p}\right]$. Then $f[R]=u_{p} \cdot C_{p}$.

Now $f^{-1}\left[c_{p}\right]$ is an ideal of $f^{-1}\left[A \cup C_{0} \cup C_{p}\right]$. So by Lemma 3.3. for any $x \in C_{p}$, $g\left(x_{\ell}\right) \cdot R$ is a minimal right ideal of $f^{-1}\left[c_{p}\right]$ and $L \cdot g\left(x_{r}\right)$ is a minimal left ideal of $f^{-1}\left[c_{p}\right]$. Therefore $\left(g\left(x_{\ell}\right) \cdot R\right) \cap\left(L \cdot g\left(x_{r}\right)\right)=g\left(x_{\ell}\right) \cdot R \cdot L \cdot g\left(x_{r}\right)$ is a group. Let $g(x)$ be the identity of $g\left(x_{\ell}\right) \cdot R \cdot L \cdot g\left(x_{r}\right)$. Notice that (iv) is satisfied. 
To verify (i), let $q \leq p$, let $x \in C_{p}$, and let $y \in A \cup C_{q}$. To see that $g(x y)=g(x)$. $g(y)$, we show that $g(x) \cdot g(y)$ is an idempotent in the same group as $g(x y)$. Since $(x y)_{\ell}=x_{\ell}$ and by Lemma 3.3, $R \cdot g(y) \subseteq R$, we have that $g(x y) \in g\left(\left(x y_{\ell}\right)\right) \cdot R=$ $g\left(x_{\ell}\right) \cdot R$ and $g(x) \cdot g(y) \in g\left(x_{\ell}\right) \cdot R \cdot g(y) \subseteq g\left(x_{\ell}\right) \cdot R$.

Also, $(x y)_{r}=y_{r}$ and so $g(x y) \in L \cdot g\left((x y)_{r}\right)=L \cdot g\left(y_{r}\right)$. To see that $g(x) \cdot g(y) \in$ $L \cdot g\left(y_{r}\right)$, we consider two cases. If $y \in C_{p}$, then $g(x) \cdot g(y) \in g(x) \cdot L \cdot g\left(y_{r}\right) \subseteq$ $L \cdot g\left(y_{r}\right)$. Now assume that $q<p$ (and $y \in A \cup C_{q}$ ). Note that $L \subseteq f^{-1}\left[c_{p}\right]$. $g\left(u_{q}\right)$; so $g\left(u_{q}\right)$ is a right identity for $L$ and thus $L=L \cdot g\left(u_{q}\right)$. Also, a simple computation establishes that $u_{q} x_{r} y=u_{q} y_{r}$. Therefore, using the fact that (i) holds at $q, g\left(u_{q}\right) \cdot g\left(x_{r}\right) \cdot g(y)=g\left(u_{q} x_{r}\right) \cdot g(y)=g\left(u_{q} x_{r} y\right)=g\left(u_{q} y_{r}\right)=g\left(u_{q}\right) \cdot g\left(y_{r}\right)$ and thus $g(x) \cdot g(y) \in L \cdot g\left(x_{r}\right) \cdot g(y)=L \cdot g\left(u_{q}\right) \cdot g\left(x_{r}\right) \cdot g(y)=L \cdot g\left(u_{q}\right) \cdot g\left(y_{r}\right)=L \cdot g\left(y_{r}\right)$.

Consequently, we have in any event that $g(x y)$ and $g(x) \cdot g(y)$ are members of the group $g\left(x_{l}\right) \cdot R \cdot L \cdot g\left(y_{r}\right)$. We show that they are equal by showing that $g(x) \cdot g(y)$ is idempotent.

Since $g\left(x_{r}\right) \cdot g(0)=g\left(x_{r} 0\right)=g(0)=g\left(y_{r} 0\right)=g\left(y_{r}\right) \cdot g(0)$ we have that $g(x)$. $g(0) \in L \cdot g\left(x_{r}\right) \cdot g(0)=L \cdot g(0)$ and because $g(x) \cdot g(y) \in L \cdot g\left(y_{r}\right)$ we have that $g(x) \cdot g(y) \cdot g(0) \in L \cdot g\left(y_{r}\right) \cdot g(0)=L \cdot g(0)$.

Now $g(x)=g\left(x_{\ell}\right) \cdot z \cdot g\left(x_{r}\right)$ for some $z \in L \cdot R$. Also $g(0) \cdot g\left(x_{\ell}\right)=g\left(0 x_{\ell}\right)=g\left(x_{\ell}\right)$ and so $g(0) \cdot g(x)=g\left(x_{\ell}\right) \cdot z \cdot g\left(x_{r}\right)=g(x)$. If $y \in C_{p}$ we have similarly that $g(0) \cdot g(y)=g(y)$, while otherwise $g(0) \cdot g(y)=g(0 y)=g(y)$ by (ii) of the induction hypothesis. We have that $g(x) \cdot g(0) \cdot g(x) \cdot g(0)=g(x) \cdot g(x) \cdot g(0)=g(x) \cdot g(0)$. So $g(x) \cdot g(0)$ is an idempotent in $L \cdot g(0)$, which is a minimal left ideal of $f^{-1}\left[c_{p}\right]$ by Lemma 3.3. Therefore $g(x) \cdot g(0)$ is a right identity for $L \cdot g(0)$ and thus $g(x)$. $g(y) \cdot g(0) \cdot g(x) \cdot g(0)=g(x) \cdot g(y) \cdot g(0)$. So

$$
\begin{aligned}
g(x) \cdot g(y) \cdot g(x) \cdot g(y) & =g(x) \cdot g(y) \cdot g(0) \cdot g(x) \cdot g(0) \cdot g(y) \\
& =g(x) \cdot g(y) \cdot g(0) \cdot g(y) \\
& =g(x) \cdot g(y) \cdot g(y)=g(x) \cdot g(y)
\end{aligned}
$$

as required.

By Lemma 3.3, $g(y) \cdot g\left(x_{\ell}\right) \cdot R \subseteq f^{-1}\left[c_{p}\right]$; so $g(y) \cdot g(x) \in g(y) \cdot g\left(x_{\ell}\right) \cdot R \cdot L \cdot g\left(x_{r}\right) \subseteq$ $L \cdot g\left(x_{r}\right)$. Also, $g(y x) \in L \cdot g\left((y x)_{r}\right)=L \cdot g\left(x_{r}\right)$ and by Lemma 3.3, $L \cdot g\left(x_{r}\right)$ is a minimal left ideal of $f^{-1}\left[c_{p}\right]$. To see that $g(y) \cdot g(x)$ is idempotent, note that $x y x=x$. So $g(x) \cdot g(y) \cdot g(x)=g(x y) \cdot g(x)=g(x y x)=g(x)$ and thus $g(y) \cdot g(x) \cdot g(y) \cdot g(x)=g(y) \cdot g(x)$, as required. This completes the verification of (i).

To verify (ii), let $x \in C_{p}$ and let $y \in A \cup C_{0}$. Pick $z \in L \cdot R$ such that $g(x)=$ $g\left(x_{\ell}\right) \cdot z \cdot g\left(x_{r}\right)$. Then $g(y) \cdot g\left(x_{\ell}\right)=g\left(y x_{\ell}\right)$. If $y \in A$, then $y x_{\ell}=x_{\ell}$ so that $g(y) \cdot g(x)=g(y) \cdot g\left(x_{\ell}\right) \cdot z \cdot g\left(x_{r}\right)=g\left(x_{\ell}\right) \cdot z \cdot g\left(x_{r}\right)=g(x)=g(y x)$. So assume that $y \in C_{0}$. Then $y x_{\ell}=(y x)_{\ell}$ and $(y x)_{r}=x_{r}$. So $g(y) \cdot g(x)=g(y) \cdot g\left(x_{\ell}\right) \cdot z \cdot g\left(x_{r}\right)=$ $g\left(y x_{\ell}\right) \cdot z \cdot g\left(x_{r}\right)=g\left((y x)_{\ell}\right) \cdot z \cdot g\left((y x)_{r}\right)$. So to see that $g(y) \cdot g(x)=g(y x)$ it suffices to recall from (i) that $g(y) \cdot g(x)$ is idempotent.

To verify (iii), let $n \in \omega$ and let $q \leq p$ such that $p=q+n$. Let $x \in C_{p}$ and let $y \in C_{q}$. If $n=0$, the conclusion follows from (i). So assume that $n>0$ so that $p$ is a successor ordinal and $p-1 \geq q$. Now $(y x)_{\ell}=y_{\ell}$; so $g(y x) \in g\left((y x)_{\ell}\right) \cdot R=g\left(y_{\ell}\right) \cdot R$. 
Recall that $R \subseteq g\left(u_{p-1}\right) \cdot f^{-1}\left[C_{p}\right]$ and consequently $R=g\left(u_{p-1}\right) \cdot R$. Thus,

$$
\begin{aligned}
g(y) \cdot g(x) & \in g(y) \cdot g\left(x_{\ell}\right) \cdot R & & \\
& =g(y) \cdot g\left(x_{\ell}\right) \cdot g\left(u_{p-1}\right) \cdot R & & \\
& =g\left(y x_{\ell}\right) \cdot g\left(u_{p-1}\right) \cdot R & & \text { by (i) at } q \\
& =g\left(y x_{\ell} u_{p-1}\right) \cdot R & & \text { by (iii) at } p-1 \\
& =g\left(y_{\ell} u_{p-1}\right) \cdot R & & \\
& =g\left(y_{\ell}\right) \cdot g\left(u_{p-1}\right) \cdot R & & \text { by (ii) at } p-1 \\
& =g\left(y_{\ell}\right) \cdot R . & &
\end{aligned}
$$

Now $g(y) \cdot g(x)$ is an idempotent in the same minimal left ideal of $f^{-1}\left[c_{p}\right]$ by (i). Since $g(y x)$ and $g(y) \cdot g(x)$ are also in the same minimal right ideal $g\left(y_{\ell}\right) \cdot R$, they must be equal. This completes the induction step.

Next, we establish (v). So assume that $T$ is compact and $\lambda$ is a successor. Then $K(T) \subseteq f^{-1}\left[C_{\lambda-1}\right]$ and so $K\left(f^{-1}\left[C_{\lambda-1}\right]\right) \subseteq K(T)$. Since $g(x) \in K\left(f^{-1}\left[C_{\lambda-1}\right]\right)$ for every $x \in C_{\lambda-1}$, (v) holds.

For each $p<\lambda$, let

$$
D_{p}=\left\{\prod_{q \in F} g\left(x_{q}\right): F \in \mathcal{P}_{f}(\lambda), p=\max F, \text { and for each } q \in F, x_{q} \in C_{q}\right\},
$$

where for each $F$, the product $\prod_{q \in F} g\left(x_{q}\right)$ is taken in increasing order of indices.

We show now by induction on $|F|$ that

$$
\begin{gathered}
\text { if } p<\lambda, y \in C_{p}, F \in \mathcal{P}_{f}(\lambda), x_{q} \in C_{q} \text { for each } q \in F, \\
\text { and } \max F \leq p \text {, then } g(y) \cdot \prod_{q \in F} g\left(x_{q}\right)=g\left(y \cdot \prod_{q \in F} x_{q}\right) .
\end{gathered}
$$

Let $r=\max F$. If $F=\{r\}$, then $g(y) \cdot g\left(x_{r}\right)=g\left(y x_{r}\right)$ by (i). So assume that $|F|>1$ and let $G=F \backslash\{r\}$. Then

$$
\begin{array}{rlrl}
g(y) \cdot \prod_{q \in F} g\left(x_{q}\right) & =g(y) \cdot \prod_{q \in G} g\left(x_{q}\right) \cdot g\left(x_{r}\right) & \\
& =g\left(y \cdot \prod_{q \in G} x_{q}\right) \cdot g\left(x_{r}\right) & & \text { by the induction hypothesis } \\
& =g\left(y \cdot \prod_{q \in G} x_{q} \cdot x_{r}\right) & & \text { by (i). }
\end{array}
$$

Now we show that each member of $D$ is idempotent. So let $F \in \mathcal{P}_{f}(\lambda)$, let $p=\max F$, and for each $q \in F$, let $x_{q} \in C_{q}$. If $F=\{p\}$, then $g\left(x_{p}\right)$ is idempotent. So assume that $|F|>1$ and let $G=F \backslash\{p\}$. Then

$$
\begin{aligned}
\prod_{q \in F} g\left(x_{q}\right) \cdot \prod_{q \in F} g\left(x_{q}\right) & =\prod_{q \in G} g\left(x_{q}\right) \cdot g\left(x_{p}\right) \cdot \prod_{q \in F} g\left(x_{q}\right) \\
& =\prod_{q \in G} g\left(x_{q}\right) \cdot g\left(x_{p} \cdot \prod_{q \in F} x_{q}\right) \\
& =\prod_{q \in G} g\left(x_{q}\right) \cdot g\left(x_{p} \cdot \prod_{q \in G} x_{q} \cdot x_{p}\right) \\
& =\prod_{q \in G} g\left(x_{q}\right) \cdot g\left(x_{p}\right) \\
& =\prod_{q \in F} g\left(x_{q}\right) .
\end{aligned}
$$

Now let $r, p<\lambda$, let $a \in D_{r}$, and let $b \in D_{p}$. We claim that $a b \in D_{r \vee p}$. We have that $a=\prod_{q \in F} g\left(x_{q}\right)$ and $b=\prod_{q \in G} g\left(y_{q}\right)$ where $\max F=r, \max G=p$, each $x_{q} \in C_{q}$ and each $y_{q} \in C_{q}$. If $r<\min G$, then $a b=\prod_{q \in F} g\left(x_{q}\right) \cdot \prod_{q \in G} g\left(y_{q}\right) \in D_{p}$. If $r \geq p$, then $a b=\prod_{q \in F \backslash\{r\}} g\left(x_{q}\right) \cdot g\left(x_{r} \cdot \prod_{q \in G} y_{q}\right) \in D_{r}$ (where the $\prod_{q \in F \backslash\{r\}} g\left(x_{q}\right)$ term is simply omitted if $F=\{r\})$. So assume that $\min G \leq r<p$, let $H=\{q \in$ $G: q \leq r\}$, and let $L=\{q \in G: g>r\}$. Then $a b=\prod_{q \in F \backslash\{r\}} g\left(x_{q}\right) \cdot g\left(x_{r}\right.$. $\left.\prod_{q \in H} y_{q}\right) \cdot \prod_{q \in L} g\left(y_{q}\right) \in D_{p}$.

Thus $D$ is a semigroup of idempotents and for each $p<\lambda, K\left(\bigcup_{q \leq p} D_{q}\right) \subseteq D_{p}$. Let $r \leq p$, let $a=\prod_{q \in F} g\left(x_{q}\right) \in D_{p}$, let $b=\prod_{q \in G} g\left(y_{q}\right) \in D_{r}$, and let $c=$ 
$\prod_{q \in H} g\left(z_{q}\right) \in D_{p}$. Then

$$
\begin{aligned}
a b c & =\prod_{q \in F \backslash\{p\}} g\left(x_{q}\right) \cdot g\left(x_{p} \cdot \prod_{q \in G} y_{q}\right) \cdot \prod_{q \in H} g\left(z_{q}\right) \\
& =\prod_{q \in F \backslash\{p\}} g\left(x_{q}\right) \cdot g\left(x_{p} \cdot \prod_{q \in G} y_{q} \cdot \prod_{q \in H} z_{q}\right) \\
& =\prod_{q \in F \backslash\{p\}} g\left(x_{q}\right) \cdot g\left(x_{p} z_{p}\right)
\end{aligned}
$$

and

$$
\begin{aligned}
a c & =\prod_{q \in F \backslash\{p\}} g\left(x_{q}\right) \cdot g\left(x_{p} \cdot \prod_{q \in H} z_{q}\right) \\
& =\prod_{q \in F \backslash\{p\}} g\left(x_{q}\right) \cdot g\left(x_{p} z_{p}\right) .
\end{aligned}
$$

So $a b c=a c$ and so each $D_{p}$ is a rectangular subsemigroup of $D$. To see that $D_{p}$ is a rectangular component of $D$, suppose that $a \in D_{p}$ and $b \in D_{q}$, where $q<p$. Then $f(b a b) \in C_{p}$ and $f(b) \in C_{q}$, and so $b a b \neq b$. To show that $D_{p} \approx V_{|A|}$ if $|A| \geq|\lambda| \geq \omega$, we observe that $C_{p}$ contains a left ideal $L$ and a right ideal $R$, each with $|A|$ elements. If $a, b \in L$, then $a b=a$ and so $g(a) g(b)=g(a)$. Thus $g[L]$ is contained in the left ideal $D_{p} g(b)$ of $D_{p}$. Similarly, $g[R]$ is contained in a right ideal of $D_{p}$. So $D_{p}$ contains a left ideal and a right ideal each with at least $|A|$ elements. They cannot have more than $|A|$ elements because for each $F \in \mathcal{P}_{f}(\lambda)$ with $p=\max F$, there are $|A|^{|F|}=|A|$ choices for $\prod_{q \in F} g\left(x_{q}\right)$. So $\left|D_{p}\right|=|A|$. Thus $D_{p} \approx L \times R \approx V_{|A|}$.

Two obvious questions are raised by Lemma 3.4 and Theorem 3.5 First, can the function $g$ constructed there be required to be continuous? Second, can the function $g$ in Theorem 3.5 be required to be a homomorphism? We shall answer both of these questions in the negative, even when the stronger requirements that $T$ and $C$ be compact and $C$ be a topological semigroup are added. We shall have need of the following lemma, whose routine proof we omit. (Recall that any successor ordinal is a compact Hausdorff space under its order topology.)

Lemma 3.6. Let $A$ be a compact space, let $\lambda$ be an ordinal, let $A \times A \times(\lambda+1)$ have the product topology, and let $A$ and $A \times A \times(\lambda+1)$ be clopen subsets of $C=C_{A, \lambda}$. Then $C$ is a compact topological semigroup and $C_{\lambda}=K(C)$.

We now show that, even for $\lambda=0$, one cannot require that $g$ be continuous. We remind the reader that an $F$-space is a completely regular space $X$ in which $\{x \in X: f(x)>0\}$ and $\{x \in X: f(x)<0\}$ are completely separated for all continuous $f: X \rightarrow \mathbb{R}$.

Theorem 3.7. There exist a nonempty set $A$, a topology on $C=C_{A, 1}$ such that $C$ is a compact topological semigroup and $A$ and $C_{0}$ are compact subsets of $C, a$ compact right topological semigroup $T$, and a continuous surjective homomorphism $f: T \rightarrow C$ such that there is no continuous homomorphism $g: C \rightarrow T$ for which $f \circ g$ is the identity on $C$. (In fact, there is no continuous injective function from $C$ to T.) 
Proof. Let $A=\beta \mathbb{N}$, let $C=\beta \mathbb{N} \cup(\beta \mathbb{N} \times \beta \mathbb{N} \times\{0\})$ with the topology given in Lemma 3.6, and let $T=\mathbb{H}_{\omega}$. Then $\mathbb{N} \cup(\mathbb{N} \times \mathbb{N} \times\{0\})$ is dense in $C=\Lambda(C)$. So there is a continuous surjective homomorphism $f: T \rightarrow C$ by Theorem [2.5,

Now suppose there is a continuous injective function $g: C \rightarrow T$. Then by Theorem 2.2 there is a continuous injective function from $C$ to $\mathbb{H} \subseteq \beta \mathbb{N}$. But this is impossible because $\beta \mathbb{N}$ is an $F$-space [3] Theorem 14.25]. So every compact subset $X$ of $\beta \mathbb{N}$ is an $F$-space, because every continuous function from $X$ to $[0,1]$ has a continuous extension to $\beta \mathbb{N}$, by the Tietze extension theorem. But $\beta \mathbb{N} \times \beta \mathbb{N}$ is not an $F$-space by $3,14 \mathrm{Q}]$.

Theorem 3.8. There exist a nonempty set $A$ with distinguished element 0 , a topology on $C=C_{A, \omega+1}$ such that $C$ is a compact topological semigroup and $A$ and $C_{p}$ are compact subsets of $C$ for each $p \leq \omega$, a compact right topological semigroup $T$, and a continuous surjective homomorphism $f: T \rightarrow C$ such that there is no homomorphism $g: C \rightarrow T$ for which $f \circ g$ is the identity on $C$.

Proof. Let $A=\{0\}$ and let $C=C_{A, \omega+1}$ with the topology given in Lemma 3.6 Let $u_{0}=0$, for $p<\omega$, let $u_{p+1}=(0,0, p)$, and let $u_{\omega}=(0,0, \omega)$. Then $C=\left\{u_{p}: p \leq \omega\right\}$ and $u_{p} u_{q}=u_{p \vee q}$ for all $p, q \leq \omega$. Topologically, $u_{\omega}$ is the only non-isolated point in $C$. Let $\left\langle v_{p}\right\rangle_{p<\omega}$ be a sequence of distinct points none of which are in $C$. Let $T=\left\{u_{p}: p<\omega\right\} \cup\left\{v_{p}: p<\omega\right\}$ and for $p, q<\omega$ define an operation on $T$ as follows:

$$
\begin{aligned}
& u_{p} u_{q}=u_{p \vee q}, \\
& u_{p} v_{q}=v_{p \vee q}, \\
& v_{p} u_{q}=v_{p} v_{q}=v_{p} .
\end{aligned}
$$

We leave it to the reader to verify that the operation is associative.

Let $T \backslash\left\{v_{0}\right\}$ be discrete, and let $T$ be the one point compactification of $T \backslash\left\{v_{0}\right\}$ (with $v_{0}$ as the point at infinity). We claim that $T$ is a right topological semigroup. Let $p<\omega$. To see that $\rho_{u_{p}}$ is continuous at $v_{0}$, let $W$ be a neighborhood of $v_{0}=\rho_{u_{p}}\left(v_{0}\right)$ and let $U=W \cap\left(\left\{u_{q}: p \leq q<\omega\right\} \cup\left\{v_{q}: q<\omega\right\}\right)$. Then $\rho_{u_{p}}[U] \subseteq W$. To see that $\rho_{v_{p}}$ is continuous at $v_{0}$, let $W$ be a neighborhood of $v_{0}=\rho_{v_{p}}\left(v_{0}\right)$ and let $U=\left\{u_{q}: p \leq q<\omega\right.$ and $\left.v_{q} \in W\right\} \cup\left\{v_{q}: v_{q} \in W\right\}$. Then $\rho_{v_{p}}[U] \subseteq W$.

Define $f: T \rightarrow C$ by $f\left(u_{p}\right)=u_{p}$ and $f\left(v_{p}\right)=u_{\omega}$ for each $p<\omega$. Then $f$ is a continuous surjective homomorphism. Suppose that $g: C \rightarrow T$ is a homomorphism for which $f \circ g$ is the identity. Then for $p<\omega, g\left(u_{p}\right)=u_{p}$, and there is some $q<\omega$ such that $g\left(u_{\omega}\right)=v_{q}$. But then, $v_{q+1}=u_{q+1} v_{q}=g\left(u_{q+1}\right) \cdot g\left(u_{\omega}\right)=g\left(u_{q+1} u_{\omega}\right)=$ $g\left(u_{\omega}\right)=v_{q}$, a contradiction.

We shall see next that we can get the function $g$ to be a homomorphism by requiring that $T$ be semitopological. This corollary can then be viewed as saying that $C$ is something like an absolute co-retract in the category of semitopological semigroups. $C$ becomes an absolute co-retract in the category of compact semitopological semigroups if it is given a topology for which it is in this category with $A$ and each $C_{p}$ being compact.

Corollary 3.9. Let $A$ be a nonempty set with distinguished element 0 , let $\lambda$ be an ordinal, and let $C=C_{A, \lambda}$. Let $T$ be a semitopological semigroup, and $f: T \rightarrow C$ be a continuous homomorphism such that $f^{-1}[A]$ is compact and $f^{-1}\left[C_{p}\right]$ is compact for every $p<\lambda$. Then there is a homomorphism $g: C \rightarrow T$ such that $f \circ g$ is the identity. 
Proof. At stage $p$ of the induction in the proof of Theorem 3.5 one has that for each $q<p, g\left(u_{q}\right) \cdot f^{-1}\left[c_{p}\right]$ is a compact right ideal of $f^{-1}\left[c_{p}\right]$. So one may choose a minimal right ideal $R$ of $f^{-1}\left[c_{p}\right]$ with $R \subseteq \bigcap_{q<p} g\left(u_{q}\right) \cdot f^{-1}\left[c_{p}\right]$ and $f[R]=u_{p} \cdot C_{p}$. Then, if $y \in C_{q}$ for some $q \leq p$ and $x \in C_{p}$, just as one showed in the verification of hypothesis (i) that $g(x) \cdot g(y) \in L \cdot g\left(y_{r}\right)$, one can show that $g(y) \cdot g(x) \in g\left(y_{l}\right) \cdot R$, so that $g(y) \cdot g(x)$ and $g(y x)$ are idempotents in the same group. If $y \in A$ and $x \in C_{p}$, then $g(y) \cdot g(x)=g(y x)$ by (ii). (We did not need to consider the case $y \in A$ separately at that point in the proof of Theorem 3.5 because the equation $u_{q} x_{r} y=u_{q} y_{r}$ was valid in any event. The corresponding equation $y x_{\ell} u_{q}=y_{\ell} u_{q}$ is not valid if $y \in A$.)

We now present some immediate consequences of Theorem 3.5, although with a bit more effort, we shall get a stronger result, namely Theorem 3.16

Corollary 3.10. Let $\kappa$ be an infinite cardinal and let $\lambda$ be an ordinal with $|\lambda| \leq \kappa$. Then $\mathbb{H}_{\kappa}$ contains a subsemigroup $D=\bigcup_{p \leq \lambda} D_{p}$ of idempotents where each $D_{p}$ is a rectangular component of $D$ isomorphic to $V_{2^{2^{\kappa}}}$ and the sequence $\left\langle D_{p}\right\rangle_{p \leq \lambda}$ is decreasing in the ordering of components, so that for each $p \leq \lambda, D_{p}=K\left(\cup_{q \leq p} D_{q}\right)$.

Proof. Let $\kappa$ have the discrete topology and let $A=\beta \kappa$. Let $C=C_{A, \lambda+1}$ and let $C$ have the topology described in Lemma 3.6. Let $T=\mathbb{H}_{\kappa}$. Since $\kappa \cup(\kappa \times \kappa \times(\lambda+1))$ is a dense subset of $C=\Lambda(C)$, by Theorem 2.5 there is a continuous surjective homomorphism $f: T \rightarrow C$, and so Theorem 3.5 applies.

Corollary 3.11. Let $S$ be an infinite cancellative discrete semigroup with cardinality $\kappa$ and let $\lambda$ be an ordinal with $|\lambda| \leq \kappa$. Then $\beta S \backslash S$ contains a subsemigroup $D=\bigcup_{p \leq \lambda} D_{p}$ of idempotents where each $D_{p}$ is a rectangular component of $D$ isomorphic to $V_{2^{2^{\kappa}}}$ and the sequence $\left\langle D_{p}\right\rangle_{p \leq \lambda}$ is decreasing in the ordering of components, so that for each $p \leq \lambda, D_{p}=K\left(\bigcup_{q \leq p} D_{q}\right)$. If $S=(\mathbb{N},+), S=(\mathbb{N}, \cdot)$, or $S$ is a countably infinite discrete group, then $D_{\lambda} \subseteq K(\beta S)$.

Proof. By Theorem 2.7, $\beta S \backslash S$ contains a topological and algebraic copy $T$ of $\mathbb{H}_{\kappa}$. (If $S=(\mathbb{N},+)$, choose $T=\mathbb{H}$. If $S=(\mathbb{N}, \cdot)$ or $S$ is a countably infinite discrete group, choose $T$ as in Theorem 2.4 or Theorem 2.3 respectively.) Then Corollary 3.10 applies.

If $S=(\mathbb{N},+), S=(\mathbb{N}, \cdot)$, or $S$ is a countably infinite discrete group, then $K(T)=K(\beta S) \cap T$. So by Theorem [3.5, with $\lambda+1$ in place of $\lambda$, we have $g\left[C_{\lambda}\right] \subseteq K(T) \subseteq K(\beta S)$ and so $D_{\lambda} \subseteq K(\beta S)$.

Corollary 3.12. Let $S$ be a countably infinite discrete group. Then there is a copy of $V_{2^{c}}$ contained in $K(\beta S)$.

We can completely characterise the semigroups of idempotents that can be embedded in $K(\beta \mathbb{N})$.

Corollary 3.13. Let $S$ be an infinite cancellative discrete semigroup with cardinality $\kappa$ and let $D$ be a semigroup of idempotents.

(i) There is a copy of $D$ in $\beta S \backslash S$ if $D$ is rectangular and $|D| \leq 2^{2^{\kappa}}$.

(ii) There is a copy of $D$ in $K(\beta \mathbb{N})$ if and only if $D$ is rectangular and $|D| \leq 2^{\mathfrak{c}}$.

Proof. Conclusion (i) and the sufficiency of (ii) follow immediately from Corollary 3.11. Assume now that $D$ is a semigroup of idempotents contained in $K(\beta \mathbb{N})$. Then $|D| \leq|\beta \mathbb{N}|=2^{\mathfrak{c}}$. Next observe that any subsemigroup of idempotents in $K(\beta S)$ 
must be rectangular. To see this, suppose that $x, y, z \in K(\beta S)$. Then $x z$ and $x y z$ belong to the same minimal left ideal and to the same minimal right ideal. Hence, if they are idempotent, they must be equal.

Recall that any two maximal groups in the smallest ideal of a compact right topological semigroup are isomorphic. We see that we can get the direct product of such groups with an embedded rectangular semigroup in the smallest ideal as well.

Theorem 3.14. Let $T$ be a compact right topological semigroup, let $D$ be a rectangular subsemigroup of $K(T)$, and let $G$ be a maximal subgroup of $K(T)$. There is an algebraic copy of $D \times G$ contained in $K(T)$.

Proof. Let $L$ be a minimal left ideal of $D$ and let $R$ be a minimal right ideal of $D$. Since $D$ is rectangular, $D$ is the internal direct product of $L$ and $R$, meaning that each element $x$ of $D$ can be written uniquely as $x=x_{L} x_{R}$ where $x_{L} \in L$ and $x_{R} \in R$. Also, $R L=R \cap L$ is a subgroup of $D$ and so, since $D$ consists of idempotents, $R L=\{e\}$ for some $e$. Then for any $x, y \in D, x_{R} y_{L}=e$. Note also that $(x y)_{L}=x_{L}$ and $(x y)_{R}=y_{R}$.

We may assume that $G=e T e$. Define $\varphi: D \times G \rightarrow K(T)$ by $\varphi(x, g)=x_{L} g x_{R}$. We claim that $\varphi$ is an injective homomorphism. Let $(x, g),(y, h) \in D \times G$. Then $\varphi((x, g)(y, h))=x_{L} g x_{R} y_{L} h y_{R}=x_{L} g e h y_{R}=(x y)_{L} g h(x y)_{R}=\varphi(x y, g h)$.

Now assume that $\varphi(x, g)=\varphi(y, h)$. Then $g=e g e=x_{R} x_{L} g x_{R} x_{L}=x_{R} y_{L} h y_{R} x_{L}$ $=e h e=h$. Also, $x_{L} T \cap y_{L} T \neq \emptyset$ and $x_{L} T$ and $y_{L} T$ are minimal right ideals of $T$; so $x_{L} T=y_{L} T$. Similarly $T x_{R}=T y_{R}$. Now $x=x_{L} x_{R} \in x_{L} T \cap T x_{R}$ and $y \in y_{L} T \cap T y_{R}$. So $x$ and $y$ are idempotents in the same group and therefore $x=y$.

Corollary 3.15. $K(\beta \mathbb{N})$ contains an algebraic copy of $V_{2^{\mathrm{c}}} \times F$, where $V_{2^{\mathrm{c}}}$ is the $2^{\mathfrak{c}} \times 2^{\mathfrak{c}}$ rectangular semigroup and $F$ is the free group on $2^{\mathfrak{c}}$ generators.

Proof. By Corollary $3.13 K(\beta \mathbb{N})$ contains a copy of the $2^{\mathfrak{c}} \times 2^{\mathfrak{c}}$ rectangular semigroup, and by [4], each maximal group in $K(\beta \mathbb{N})$ contains a copy of the free group on $2^{\mathfrak{c}}$ generators. Therefore the result follows from Theorem 3.14

We now present a strengthening of Corollary 3.10, producing a longer chain of rectangular components. Recall that the Souslin number $S(X)$ of a topological space $X$ (also known as the cellularity of $X$ ) is the least cardinal $\gamma$ such that $X$ does not have a collection of $\gamma$ pairwise disjoint nonempty open subsets. See [2, Chapter 12] for considerable information about the Souslin number of the space $U(\kappa)$ of uniform ultrafilters on $\kappa$. Recall, in particular, that the Souslin number of $\mathbb{N}^{*}=\beta \mathbb{N} \backslash \mathbb{N}=U(\mathbb{N})$ is $\mathfrak{c}^{+}$.

Theorem 3.16. Let $\kappa$ be an infinite cardinal and let $\lambda$ be an infinite ordinal for which $|\lambda|<S(U(\kappa))$. There exist a set $A$ with $|A|=2^{2^{\kappa}}$ and an injection $g$ : $C_{A, \lambda} \rightarrow \mathbb{H}_{\kappa}$ such that if $q<p<\lambda, y \in C_{q}$, and $x \in C_{p}$, then $g(x) \cdot g(y)=g(x y)$, and if $p=q+n$ for some $n<\omega$, then $g(y) \cdot g(x)=g(y x)$. Also, $\mathbb{H}_{\kappa}$ contains a semigroup $D=\bigcup_{p<\lambda} D_{p}$ of idempotents where for each $p<\lambda, D_{p}$ is a rectangular component of $D$ isomorphic to $V_{2^{2^{\kappa}}}, g\left[C_{p}\right] \subseteq D_{p}$, and the sequence $\left\langle D_{p}\right\rangle_{p<\lambda}$ is decreasing in the ordering of components, so that for each $p<\lambda, D_{p}=K\left(\bigcup_{q \leq p} D_{q}\right)$. If $\lambda$ is a successor, then $D_{\lambda-1} \subseteq K\left(\mathbb{H}_{\kappa}\right)$.

Proof. Since $|\lambda|<S(U(\kappa))$, choose a family $\left\langle E_{p}\right\rangle_{p<\lambda}$ of subsets of $\kappa$ such that each $\left|E_{p}\right|=\kappa$ and $\left|E_{p} \cap E_{q}\right|<\kappa$ when $p \neq q$. For each $p<\lambda$ we define $\phi_{p}: W_{\kappa} \rightarrow W_{\kappa}$ 
by $\phi_{p}(w)=\sum_{\alpha \in E_{p} \cap \operatorname{supp}(w)} e_{\alpha}\left(\right.$ where $\left.\sum_{\alpha \in \emptyset} e_{\alpha}=0\right)$ and let $\widetilde{\phi_{p}}: \beta W_{\kappa} \rightarrow \beta W_{\kappa}$ be the continuous extension of $\phi_{p}$. If $v, w \in W_{\kappa}$ and $\operatorname{supp}(v) \cap \operatorname{supp}(w)=\emptyset$, then $\phi_{p}(v+w)=\phi_{p}(v)+\phi_{p}(w)$. So by [6. Theorem 4.21] the restriction of $\widetilde{\phi}_{p}$ to $\mathbb{H}_{\kappa}$ is a homomorphism.

Next observe that for $x \in \mathbb{H}_{\kappa}, \widetilde{\phi_{p}}(x) \in\{0\} \cup \mathbb{H}_{\kappa}$. If there exist $B \in x$ and $\alpha<\kappa$ such that $\operatorname{supp}(w) \cap E_{p}=\emptyset$ whenever $w \in B$ and $\min \operatorname{supp}(w) \geq \alpha$, then $\widetilde{\phi_{p}}(x)=0$ because $\phi_{p}$ is constantly 0 on $\{w \in B: \min \operatorname{supp}(w) \geq \alpha\} \in x$. Otherwise $\left\{\phi_{p}[\{w \in B: \min \operatorname{supp}(w) \geq \alpha\}]: B \in x\right.$ and $\left.\alpha<\kappa\right\}$ has the finite intersection property and so is contained in an ultrafilter $y$. This $y \in \mathbb{H}_{\kappa}$ and $y=\widetilde{\phi_{p}}(x)$.

Let $T_{0}=\mathbb{H}_{\kappa} \cap c \ell\left\{w \in W_{\kappa}: \operatorname{supp}(w) \subseteq E_{0}\right\}$. Notice that $T_{0}$ is a compact subsemigroup of $\mathbb{H}_{\kappa}$. For each $p$ with $0<p<\lambda$ let

$$
T_{p}=\left\{x \in \mathbb{H}_{\kappa}: \widetilde{\phi_{p}}(x) \in \mathbb{H}_{\kappa} \text { and for all } q \text { with } p<q \leq \lambda, \widetilde{\phi_{q}}(x)=0\right\} .
$$

To see that $T_{p} \neq \emptyset$, let $x$ be a uniform ultrafilter on $\left\{e_{\alpha}: \alpha \in E_{p}\right\}$. If $q \neq p$, then $\left|E_{q} \cap E_{p}\right|<\kappa$. So $\widetilde{\phi_{q}}(x)=0$, while $\widetilde{\phi_{p}}(x)=x \in \mathbb{H}_{\kappa}$ (because $\phi_{p}$ is the identity on $\left.\left\{e_{\alpha}: \alpha \in E_{p}\right\}\right)$. Since $\widetilde{\phi_{q}}$ is a homomorphism on $\mathbb{H}_{\kappa}$ for each $q \leq \lambda$ we have that $T_{p}$ is a subsemigroup of $\mathbb{H}_{\kappa}$. Since $T_{p}=\mathbb{H}_{\kappa} \cap{\widetilde{\phi_{p}}}^{-1}\left[\mathbb{H}_{\kappa}\right] \cap \bigcap_{p<q \leq \lambda}{\widetilde{\phi_{q}}}^{-1}[\{0\}], T_{p}$ is compact.

If $\lambda$ is a successor, let $T_{\lambda-1}=\mathbb{H}_{\kappa} \cap \bigcap_{p<\lambda}{\widetilde{\phi_{p}}}^{-1}\left[\mathbb{H}_{\kappa}\right]$. Then $T_{\lambda-1}$ is clearly a compact subsemigroup of $\mathbb{H}_{\kappa}$ provided $T_{\lambda-1} \neq \emptyset$. We show in fact that $K\left(\mathbb{H}_{\kappa}\right) \subseteq$ $T_{\lambda-1}$. Let $x \in K\left(\mathbb{H}_{\kappa}\right)$, let $p<\lambda$, and let $y$ be a uniform ultrafilter on $\left\{e_{\alpha}\right.$ : $\left.\alpha \in E_{p}\right\}$. By [6] Theorem 4.39] pick $z \in \mathbb{H}_{\kappa}$ such that $x=z+y+x$. Then $\widetilde{\phi_{p}}(x)=\widetilde{\phi_{p}}(z)+\widetilde{\phi_{p}}(y)+\widetilde{\phi_{p}}(x)=\widetilde{\phi_{p}}(z)+y+\widetilde{\phi_{p}}(x) \neq 0$.

Next observe that for $p, q<\lambda, T_{p}+T_{q}=T_{p \vee q}$ and if $p \neq q$, then $T_{p} \cap T_{q}=\emptyset$.

Let $T=\bigcup_{p<\lambda} T_{p}$. If $T$ has the relative topology induced by $\mathbb{H}, T$ is a right topological semigroup.

Let $A=\mathbb{H}_{\kappa} \cap c \ell\left\{e_{\alpha}: \alpha \in E_{0}\right\}$. Then $A$ is exactly the set of uniform ultrafilters on $\left\{e_{\alpha}: \alpha \in E_{0}\right\}$, and so $|A|=2^{2^{\kappa}}$. Let $C=C_{A, \lambda}$.

We shall now construct a surjective homomorphism $f: T \rightarrow C$. We first introduce some mappings. Let $\theta: W_{\kappa} \rightarrow\left\{e_{\alpha}: \alpha \in E_{0}\right\}$ be a function whose restriction to $\left\{e_{\alpha}: \alpha \in E_{0}\right\}$ is the identity, whose restriction to $\left\{e_{\alpha}: \alpha \in E_{1}\right\}$ is a bijection, and whose restriction to $W_{\kappa} \backslash\left\{e_{\alpha}: \alpha \in E_{0} \cup E_{1}\right\}$ is a bijection. (In particular, $\theta$ is at most three-to-one.) Let $\widetilde{\theta}: \beta W_{\kappa} \rightarrow c \ell\left\{e_{\alpha}: \alpha \in E_{0}\right\}$ be the continuous extension of $\theta$.

Let $\epsilon(0)=\delta(0)=0$. For $w \in W_{\kappa} \backslash\{0\}$, let $\epsilon(w)=e_{\gamma}$ where $\gamma=\max \operatorname{supp}(w)$. If $\operatorname{supp}(w) \subseteq E_{0}$, let $\delta(w)=0$. Otherwise let $\delta(w)=e_{\alpha}$ where $\alpha=\min \left(\operatorname{supp}(w) \backslash E_{0}\right)$. Let $\widetilde{\delta}: \beta W_{\kappa} \rightarrow\{0\} \cup c \ell\left\{e_{\alpha}: \alpha<\kappa\right\}$ and $\widetilde{\epsilon}: \beta W_{\kappa} \rightarrow\{0\} \cup c \ell\left\{e_{\alpha}: \alpha<\kappa\right\}$ be the continuous extensions of $\delta$ and $\epsilon$ respectively. Notice that $\delta$ is the identity on $\left\{e_{\alpha}: \alpha \in \kappa \backslash E_{0}\right\}$ and $\epsilon$ is the identity on $\left\{e_{\alpha}: \alpha<\kappa\right\}$. So $\widetilde{\delta}$ is the identity on $\mathbb{H}_{\kappa} \cap c \ell\left\{e_{\alpha}: \alpha \in \kappa \backslash E_{0}\right\}$ and $\widetilde{\epsilon}$ is the identity on $\mathbb{H}_{\kappa} \cap c \ell\left\{e_{\alpha}: \alpha<\kappa\right\}$. We claim that for $x, y \in \mathbb{H}_{\kappa}$,

$$
\begin{aligned}
& \widetilde{\epsilon}(x+y)=\widetilde{\epsilon}(y), \\
& \widetilde{\delta}(x+y)= \begin{cases}\widetilde{\delta}(x) & \text { if } x \notin T_{0}, \\
\widetilde{\delta}(y) & \text { if } x \in T_{0}\end{cases}
\end{aligned}
$$


For $w, v \in W_{\kappa}$, if $\max \operatorname{supp}(v)>\max \operatorname{supp}(w)$, then $\epsilon(w+v)=\epsilon(v)$ so that $\widetilde{\epsilon}(w+y)=\widetilde{\epsilon}(y)$; if $\operatorname{supp}(w) \subseteq E_{0}$, then $\delta(w+v)=\delta(v)$ so that $\widetilde{\delta}(w+y)=\widetilde{\delta}(y)$. For $w, v \in W_{\kappa}$, if $\max \operatorname{supp}(w)<\min \operatorname{supp}(v)$ and $\operatorname{supp}(w) \backslash E_{0} \neq \emptyset$, then $\delta(w+v)=$ $\delta(w)$; so $\widetilde{\delta}(w+y)=\delta(w)$. The equations in $(*)$ then follow by the continuity of $\widetilde{\delta} \circ \rho_{y}$ and $\widetilde{\epsilon} \circ \rho_{y}$.

For $x \in T_{0}$, let $f(x)=\widetilde{\theta}(\widetilde{\epsilon}(x))$. If $0<p<\lambda$ and $x \in T_{p}$, let

$$
f(x)= \begin{cases}(\widetilde{\theta}(\widetilde{\delta}(x)), \widetilde{\theta}(\widetilde{\epsilon}(x)), p-1) & \text { if } p<\omega, \\ (\widetilde{\theta}(\widetilde{\delta}(x)), \widetilde{\theta}(\widetilde{\epsilon}(x)), p) & \text { if } p \geq \omega .\end{cases}
$$

For any $x \in \mathbb{H}_{\kappa}$, one has $\widetilde{\theta}(\widetilde{\delta}(x)) \in A$ and $\widetilde{\theta}(\widetilde{\epsilon}(x)) \in A$. (We have that $\widetilde{\theta}\left[\mathbb{H}_{\kappa}\right] \subseteq \mathbb{H}_{\kappa}$ because $\theta$ is at most three-to-one.) Thus $f\left[T_{0}\right] \subseteq A, f\left[T_{p}\right] \subseteq C_{p-1}$ if $0<p<\omega$, and $f\left[T_{p}\right] \subseteq C_{p}$ if $\omega \leq p<\lambda$.

Given $x \in A \subseteq T_{0}$, one has $f(x)=\tilde{\theta}(\widetilde{\epsilon}(x))=\tilde{\theta}(x)=x$; so $f\left[T_{0}\right]=A$. Now let $p<\lambda$ and let $(y, z, p) \in C_{p}$. If $p<\omega$, let $q=p+1$; otherwise let $q=p$. Pick $y^{\prime} \in c \ell\left\{e_{\alpha}: \alpha \in E_{1}\right\}$ such that $\widetilde{\theta}\left(y^{\prime}\right)=y$. Pick $x \in T_{q}$. Then $y^{\prime}+x+z \in T_{q}$ and

$$
\begin{aligned}
f\left(y^{\prime}+x+z\right) & =\left(\widetilde{\theta}\left(\widetilde{\delta}\left(y^{\prime}+x+z\right)\right), \widetilde{\theta}\left(\widetilde{\epsilon}\left(y^{\prime}+x+z\right)\right), p\right) \\
& =\left(\widetilde{\theta}\left(\widetilde{\delta}\left(y^{\prime}\right)\right), \widetilde{\theta}(\widetilde{\epsilon}(z)), p\right) \\
& =\left(\widetilde{\theta}\left(y^{\prime}\right), \widetilde{\theta}(z), p\right) \\
& =(y, z, p) .
\end{aligned}
$$

Therefore, $f\left[T_{q}\right]=C_{p}$.

The verification that $f$ is a homomorphism is routine using the equations $(*)$.

Choose $g: C \rightarrow T$ and $\left\langle D_{p}\right\rangle_{p<\lambda}$ as guaranteed by Theorem 3.5 Since we have already observed that $|A|=2^{2^{\kappa}}$, all conclusions follow immediately except the assertion that $D_{\lambda-1} \subseteq K\left(\mathbb{H}_{\kappa}\right)$ when $\lambda$ is a successor. To see this recall that $K\left(\mathbb{H}_{\kappa}\right) \subseteq T_{\lambda-1}$ so that $K\left(\mathbb{H}_{\kappa}\right)$ is an ideal of $T_{\lambda-1}$ and thus $K\left(T_{\lambda-1}\right) \subseteq K\left(\mathbb{H}_{\kappa}\right)$. By Theorem 3.5 (iv), $g\left[C_{\lambda-1}\right] \subseteq K\left(f^{-1}\left[C_{\lambda-1}\right]\right)=K\left(T_{\lambda-1}\right)$. So $D \cap K\left(\mathbb{H}_{\kappa}\right) \neq \emptyset$ and is thus an ideal of $D$ and therefore $D_{\lambda-1}=K(D) \subseteq D \cap K\left(\mathbb{H}_{\kappa}\right)$.

Corollary 3.17. Let $\lambda$ be an ordinal for which $|\lambda|=\mathfrak{c}$. There exist a set $A$ with $|A|=2^{\mathfrak{c}}$ and an injection $g: C_{A, \lambda} \rightarrow \mathbb{H}$ such that if $q<p<\lambda, y \in C_{q}$, and $x \in C_{p}$, then $g(x) \cdot g(y)=g(x y)$, and if $p=q+n$ for some $n<\omega$, then $g(y) \cdot g(x)=g(y x)$. Also, $\mathbb{H}$ contains a semigroup $D=\bigcup_{p<\lambda} D_{p}$ of idempotents where for each $p<\lambda$, $D_{p}$ is a rectangular component of $D, g\left[C_{p}\right] \subseteq D_{p}$, and the sequence $\left\langle D_{p}\right\rangle_{p<\lambda}$ is decreasing in the ordering of components. For each $p<\lambda,\left|D_{p}\right|=2^{\mathfrak{c}}$ and if $\lambda$ is a successor, then $D_{\lambda-1} \subseteq K(\mathbb{H}) \subseteq K(\beta \mathbb{N})$.

Proof. By Theorem $2.2 \mathbb{H}$ and $\mathbb{H}_{\omega}$ are topologically and algebraically isomorphic. Also $S(U(\omega))=c^{+}$. So this is an immediate consequence of Theorem 3.16

It was shown in [5. Corollary 3.4] that there is a $\leq_{L^{-}}$-chain $\left\langle u_{\sigma}\right\rangle_{\sigma<\omega_{1}}$ of distinct idempotents in $\beta \mathbb{N}$ with the property that for each $\sigma<\omega_{1}, u_{\sigma+1} \leq u_{\sigma}$. We are now able to establish a considerably stronger statement. (The necessity in the following corollary was also established in [5], but we include the short proof for completeness.)

Corollary 3.18. Let $\lambda$ be an ordinal. There is $a \leq_{L}$-chain $\left\langle u_{\sigma}\right\rangle_{\sigma<\lambda}$ of distinct idempotents in $\beta \mathbb{N}$ with the property that for each $\sigma<\lambda, u_{\sigma+1} \leq u_{\sigma}$ if and only 
if $|\lambda| \leq \mathfrak{c}$. If $|\lambda| \leq \mathfrak{c}$ and $\lambda$ is a successor, one may choose such a sequence with $u_{\lambda-1} \in K(\beta \mathbb{N})$.

Proof. Necessity. For each $\sigma<\lambda, \mathbb{N}^{*}+u_{\sigma}$ properly contains the compact set $\mathbb{N}^{*}+u_{\sigma+1}$. So one can choose a clopen subset $U_{\sigma}$ of $\beta \mathbb{N}$ with $\mathbb{N}^{*}+u_{\sigma+1} \subseteq U_{\sigma}$ and $\left(\mathbb{N}^{*}+u_{\sigma}\right) \backslash U_{\sigma} \neq \emptyset$. The clopen subsets of $\beta \mathbb{N}$ correspond exactly to the subsets of $\mathbb{N}$ and so there are exactly $\mathfrak{c}$ of them.

Sufficiency. Choose $A$ and $g$ as guaranteed by Corollary 3.17 for $\lambda$. For each $p<\lambda$, let $u_{p}=g(0,0, p)$. If $\lambda$ is a successor, then $u_{\lambda-1} \in g\left[C_{\lambda-1}\right] \subseteq D_{\lambda-1} \subseteq$ $K(\beta \mathbb{N})$.

Question 3.19. Is there a decreasing $\leq$-chain of idempotents in $\mathbb{N}^{*}$ indexed by $\omega+1$ ?

We close this section by observing that it is consistent with ZFC that there are idempotents in $\beta \mathbb{N}$ that are not members of any nontrivial rectangular subsemigroup of $\beta \mathbb{N}$. Indeed, by [6, Theorems 12.19 and 12.29 and Lemma 12.44], Martin's Axiom implies that there is an idempotent $p \in \beta \mathbb{N}$ such that, whenever $q \in \beta \mathbb{N}$, $r \in \bigcap_{n=1}^{\infty} c l_{\beta \mathbb{N}}(\mathbb{N} n)$, and $p=q+r$, one must have $p=q=r$. In particular, if $p=p+q+p$, then $p=q$.

It can be shown in ZFC that there are idempotents $p$ in $\beta \mathbb{N}$ that are strongly right maximal; i.e., the equation $q+p=p$, with $q \in \beta \mathbb{N}$, implies that $q=p$ [6, Theorem 9.10]. If $p$ is an idempotent of this kind, $p$ does not belong to any semigroup in $\beta \mathbb{N}$ isomorphic to a semigroup of the form $V_{|A|}$ unless $|A|=1$.

\section{Chains of Rectangular Semigroups as CO-Retracts}

It was shown in [10] that certain infinite chains of finite rectangular semigroups are absolute co-retracts. We prove in this section a similar theorem in which the the rectangular semigroups are allowed to be infinite. As a consequence, we obtain additional semigroups which can be algebraically embedded in $\mathbb{H}_{\kappa}$.

Definition 4.1. Let $\mathcal{A}=\left\langle A_{n}\right\rangle_{n<\omega}$ and $\mathcal{B}=\left\langle B_{n}\right\rangle_{n<\omega}$ be sequences of sets. Assume that each $A_{n}$ has a designated element $\alpha_{n}$ and each $B_{n}$ has a designated element $\delta_{n}$. Suppose also that, for each $n<\omega$, either $A_{n}=\left\{\alpha_{n}\right\}$ or $B_{n}=\left\{\delta_{n}\right\}$. For each $p<\omega$, we define $D_{p}$ to be the set of pairs of words of the form $\left(a_{0} a_{1} \cdots a_{p}, b_{p} b_{p-1} \cdots b_{0}\right)$, where $a_{i} \in A_{i}$ and $b_{i} \in B_{i}$ for each $i \in\{0,1, \ldots, p\}$. For $0<\lambda \leq \omega$, we let $D_{\mathcal{A}, \mathcal{B}, \lambda}=\bigcup_{p<\lambda} D_{p}$. We define a semigroup operation on $D_{\mathcal{A}, \mathcal{B}, \lambda}$ as follows: if $x=\left(a_{0} a_{1} \cdots a_{p}, b_{p} b_{p-1} \cdots b_{0}\right) \in D_{p}$ and $y=\left(c_{0} c_{1} \cdots c_{q}, d_{q} d_{q-1} \cdots d_{0}\right) \in D_{q}$, where $q \leq p$, then

$$
x y=\left(a_{0} a_{1} \cdots a_{p}, b_{p} \cdots b_{q+1} d_{q} d_{q-1} \cdots d_{0}\right)
$$

and

$$
y x=\left(c_{0} c_{1} \cdots c_{q} a_{q+1} \cdots a_{p}, b_{p} b_{p-1} \cdots b_{0}\right) .
$$

We leave the verification that the operation is associative to the reader. Observe that each $D_{p}$ is a rectangular semigroup.

Notice that if $A$ is a set with designated element $0, A_{0}=\{0\}, B_{0}=A, A_{1}=A$, $B_{1}=\{0\}$, and $A_{n}=B_{n}=\{0\}$ for $n>1$, then $D_{\mathcal{A}, \mathcal{B}, \omega}$ is isomorphic to $C_{A, \omega}$. (Send $(0, a)$ to $a$ and for $p>0$ send the element $(0 a 00 \cdots 0,00 \cdots 0 b)$ of $D_{p}$ to $(a, b, p-1)$.) Thus the structure of $D_{\mathcal{A}, \mathcal{B}, \omega}$ is, in general, considerably more complicated than that of $C_{A, \omega}$. 
Definition 4.2. Let $p<\omega$ and let $x=\left(a_{0} a_{1} \cdots a_{p}, b_{p} b_{p-1} \cdots b_{0}\right) \in D_{p}$. We define elements $\phi_{1}(x)$ and $\phi_{2}(x)$ in $D_{p}$ by $\phi_{1}(x)=\left(\alpha_{0} \alpha_{1} \cdots \alpha_{p-1} a_{p}, \delta_{p} \delta_{p-1} \cdots \delta_{0}\right)$ and $\phi_{2}(x)=\left(\alpha_{0} \alpha_{1} \cdots \alpha_{p}, b_{p} \delta_{p-1} \delta_{p-2} \cdots \delta_{0}\right)$ and if $p>0$, we define $x_{\ell}$ and $x_{r}$ in $D_{p-1}$ by $x_{\ell}=\left(a_{0} a_{1} \cdots a_{p-1}, \delta_{p-1} \delta_{p-2} \cdots \delta_{0}\right)$ and $x_{r}=\left(\alpha_{0} \alpha_{1} \cdots \alpha_{p-1}, b_{p-1} b_{p-2} \cdots b_{0}\right)$. We put $u_{p}=\left(\alpha_{0} \alpha_{1} \cdots \alpha_{p}, \delta_{p} \delta_{p-1} \cdots \delta_{0}\right) \in D_{p}$.

We show that $D$ is something like an absolute co-retract.

Theorem 4.3. Let $\mathcal{A}=\left\langle A_{n}\right\rangle_{n<\omega}$ and $\mathcal{B}=\left\langle B_{n}\right\rangle_{n<\omega}$ be sequences of sets as in Definition 4.1. let $0<\lambda \leq \omega$, and let $D=D_{\mathcal{A}, \mathcal{B}, \lambda}$. Let $T$ be a right topological semigroup, and let $f: T \rightarrow D$ be a surjective homomorphism such that $f^{-1}\left[D_{p}\right]$ is compact for each $p<\lambda$. Then there is a homomorphism $g: D \rightarrow T$ for which $f \circ g$ is the identity. If $T$ is compact and $\lambda<\omega$, then $g\left[D_{\lambda-1}\right] \subseteq K(T)$.

Proof. We may assume that $A_{0}=\left\{\alpha_{0}\right\}$ so that $D_{0}$ is a right zero semigroup. Exactly as in the first paragraph of the proof of Lemma 3.4 we can define $g: D_{0} \rightarrow T$ such that $g$ is a homomorphism and $f \circ g$ is the identity on $D_{0}$. So we assume that $p>0$ and $g$ has been defined on $\bigcup_{q<p} D_{q}$.

For each $x \in D_{p}$, note that $x D_{p}$ is a minimal right ideal of $D_{p}$ and $D_{p} x$ is a minimal left ideal of $D_{p}$. So we may choose a minimal right ideal $R(x)$ of $f^{-1}\left[D_{p}\right]$ and a minimal left ideal $L(x)$ of $f^{-1}\left[D_{p}\right]$ such that $f[R(x)]=x D_{p}$ and $f[L(x)]=$ $D_{p} x$. Given $x \in D_{p}$, we have by Lemma 3.3 that $g\left(x_{\ell}\right) R\left(\phi_{1}(x)\right)$ is a minimal right ideal of $f^{-1}\left[D_{p}\right]$ and $L\left(\phi_{2}(x)\right) g\left(x_{r}\right)$ is a minimal left ideal of $f^{-1}\left[D_{p}\right]$. So we may define $g(x)$ to be the identity of the group $g\left(x_{\ell}\right) R\left(\phi_{1}(x)\right) L\left(\phi_{2}(x)\right) g\left(x_{r}\right)$. Notice that if $T$ has a smallest ideal (in particular if $T$ is compact) and $\lambda=p+1$, then $K(T) \subseteq f^{-1}\left[D_{p}\right]$. So $K\left(f^{-1}\left[D_{p}\right]\right) \subseteq K(T)$ and thus $g\left[D_{p}\right] \subseteq K(T)$.

Now $f(g(x)) \in x_{\ell} \phi_{1}(x) D_{p}=x D_{p}$ and $f(g(x)) \in D_{p} \phi_{2}(x) x_{r}=D_{p} x$. So $f(g(x))$ is an idempotent in $x D_{p} x$ and thus $f(g(x))=x$.

Suppose that $x \in D_{p}$ and $y \in D_{q}$ where $q \leq p$. Then $\phi_{1}(x)=\phi_{1}(x y)$ and $x_{\ell}=(x y)_{\ell}$. So $g(x y) \in g\left(x_{\ell}\right) R\left(\phi_{1}(x)\right)$ and $g(x) g(y) \in g\left(x_{\ell}\right) R\left(\phi_{1}(x)\right) g(y) \subseteq$ $g\left(x_{\ell}\right) R\left(\phi_{1}(x)\right)$ by Lemma 3.3. Therefore, $g(x y)$ and $g(x) g(y)$ are members of the same minimal right ideal of $f^{-1}\left[D_{p}\right]$.

If $q<p, \phi_{2}(x y)=\phi_{2}(x)$ and $(x y)_{r}=x_{r} y$. So $g(x y) \in L\left(\phi_{2}(x)\right) g\left((x y)_{r}\right)$ and $g(x) g(y) \in L\left(\phi_{2}(x)\right) g\left(x_{r}\right) g(y)=L\left(\phi_{2}(x)\right) g\left(x_{r} y\right)=L\left(\phi_{2}(x)\right) g\left((x y)_{r}\right)$.

If $q=p, \phi_{2}(x y)=\phi_{2}(y)$ and $(x y)_{r}=y_{r}$. So $g(x y) \in L\left(\phi_{2}(y)\right) g\left(y_{r}\right)$ and $g(x) g(y) \in g(x) L\left(\phi_{2}(y)\right) g\left(y_{r}\right)$. Thus in any event $g(x y)$ and $g(x) g(y)$ are in the same minimal left ideal of $f^{-1}\left[D_{p}\right]$.

By a left-right switch of the above arguments we have that $g(y x)$ and $g(y) g(x)$ are in the same minimal left ideal and the same minimal right ideal of $f^{-1}\left[D_{p}\right]$.

First assume that $q<p$. Pick $a \in R\left(\phi_{1}(x)\right) L\left(\phi_{2}(x)\right)$ such that $g(x)=$ $g\left(x_{\ell}\right) \operatorname{ag}\left(x_{r}\right)$. Then

$$
\begin{aligned}
g(x) g(y) g(x) & =g\left(x_{\ell}\right) \operatorname{ag}\left(x_{r}\right) g(y) g\left(x_{\ell}\right) \operatorname{ag}\left(x_{r}\right) \\
& =g\left(x_{\ell}\right) \operatorname{ag}\left(x_{r} y x_{\ell}\right) \operatorname{ag}\left(x_{r}\right) \\
& =g\left(x_{\ell}\right) \operatorname{ag}\left(x_{r} x_{\ell}\right) \operatorname{ag}\left(x_{r}\right) \\
& =g\left(x_{\ell}\right) \operatorname{ag}\left(x_{r}\right) g\left(x_{\ell}\right) \operatorname{ag}\left(x_{r}\right)=g(x) g(x)=g(x) .
\end{aligned}
$$

So $g(x) g(y) g(x) g(y)=g(x) g(y)$ and $g(y) g(x) g(y) g(x)=g(y) g(x)$ and thus $g(x) g(y)$ $=g(x y)$ and $g(y) g(x)=g(y x)$.

Now assume that $q=p$. Assume also that $B_{p}=\left\{\delta_{p}\right\}$. (The case that $A_{p}=\left\{\alpha_{p}\right\}$ then proceeds by a left-right switch of the following argument.) Then $\phi_{2}(x)=$ 
$\phi_{2}(y)=u_{p}$. Also $x_{r} y_{\ell}=y_{r} x_{\ell}=u_{p-1}$. Thus $g(x) g\left(y_{\ell}\right) \in L\left(\phi_{2}(x)\right) g\left(x_{r}\right) g\left(y_{\ell}\right)=$ $L\left(u_{p}\right) g\left(u_{p-1}\right)$ and $g(y) g\left(x_{\ell}\right) \in L\left(u_{p}\right) g\left(u_{p-1}\right)$, which is a minimal left ideal of $f^{-1}\left[D_{p}\right]$ by Lemma [3.3. We have already verified that $g(x) g\left(y_{\ell}\right)$ and $g(y) g\left(x_{\ell}\right)$ are idempotents. So, since they are idempotents in the same minimal left ideal $g(x) g\left(y_{\ell}\right) g(y) g\left(x_{\ell}\right)=g(x) g\left(y_{\ell}\right)$, we have

$$
\begin{aligned}
g(x) g(y) g(x) & =g(x) g\left(y_{\ell} y\right) g\left(x_{\ell} x\right) \\
& =g(x) g\left(y_{\ell}\right) g(y) g\left(x_{\ell}\right) g(x) \\
& =g(x) g\left(y_{\ell}\right) g(x) \\
& =g\left(x x_{r}\right) g\left(y_{\ell}\right) g\left(x_{\ell} x\right) \\
& =g(x) g\left(x_{r} y_{\ell} x_{\ell}\right) g(x) \\
& =g(x) g\left(x_{r} x_{\ell}\right) g(x) \\
& =g\left(x x_{r}\right) g\left(x_{\ell} x\right)=g(x) g(x)=g(x) .
\end{aligned}
$$

Consequently, $g(x) g(y)$ and $g(y) g(x)$ are idempotents.

Corollary 4.4. Suppose that $\kappa$ is an infinite cardinal and that each $A_{n}$ or $B_{n}$ is either $\{0\}$ or $2^{2^{\kappa}}$. Then $D_{\mathcal{A}, \mathcal{B}, \omega}$ can be embedded in $\mathbb{H}_{\kappa}$.

Proof. For each $p<\omega$, we give $D_{p}$ the topology defined by regarding $D_{p}$ as a subspace of $(\beta \kappa)^{2 p+2}$. We define the topology of $D$ by taking each $D_{p}$ to be clopen in $D$. Then $D$ is a topological semigroup with a dense subspace of cardinality $\kappa$. The conclusion then follows from Theorems 4.3 and 2.5

\section{REFERENCES}

1. J. Berglund, H. Junghenn, and P. Milnes, Analysis on semigroups, Wiley, N.Y., 1989. MR 91b:43001

2. W. Comfort and S. Negrepontis, The theory of ultrafilters, Springer-Verlag, Berlin, 1974. MR 53:135

3. L. Gillman and M. Jerison, Rings of continuous functions, van Nostrand, Princeton, 1960. MR 22:6994

4. N. Hindman and J. Pym, Free groups and semigroups in $\beta \mathbb{N}$, Semigroup Forum 30 (1984), 177-193. MR 86c:22002

5. N. Hindman and D. Strauss, Chains of idempotents in $\beta \mathbb{N}$, Proc. Amer. Math. Soc. 123 (1995), 3881-3888. MR 96b:54037

6. N. Hindman and D. Strauss, Algebra in the Stone-Čech compactification: Theory and applications, de Gruyter, Berlin, 1998. MR 99j:54001

7. D. McLean, Idempotent semigroups, Amer. Math. Monthly 61 (1954), 110-113. MR 15:681a

8. J. Pym, Semigroup structure in Stone-Čech compactifications, J. London Math. Soc. 36 (1987), 421-428. MR 89b:54043

9. W. Ruppert, Rechstopologische Halbgruppen, J. Reine Angew. Math. 261 (1973), 123-133. MR 47:6933

10. Y. Zelenyuk, On subsemigroups of $\beta \mathbb{N}$ and absolute coretracts, Semigroup Forum 63 (2001), 457-465. MR 2002f:22005

Department of Mathematics, Howard University, Washington, DC 20059

E-mail address: nhindman@aol.com

URL: http://members .aol.com/nhindman/

Department of Pure Mathematics, University of Hull, Hull hu6 7RX, United KingDOM

E-mail address: d.strauss@maths.hull.ac.uk

Faculty of Cybernetics, Kyiv Taras Shevchenko University, Volodymyrska Street 64, 01033 KYIV, UKRAINE

E-mail address: grishko@i.com.ua 